\title{
A PROJECTION-BASED ERROR ANALYSIS OF HDG METHODS FOR TIMOSHENKO BEAMS
}

\author{
FATIH CELIKER, BERNARDO COCKBURN, AND KE SHI
}

\begin{abstract}
In this paper, we give the first a priori error analysis of the hybridizable discontinuous Galerkin (HDG) methods for Timoshenko beams. The analysis is based on the use of a projection especially designed to fit the structure of the numerical traces of the HDG method. This property allows us to prove in a very concise manner that the projection of the errors is bounded in terms of the distance between the exact solution and its projection. The study of the influence of the stabilization function on the approximation is then reduced to the study of how they affect the approximation properties of the projection in a single element. Surprisingly, and unlike any other discontinuous Galerkin method, this can be done without assuming any positivity property of the stabilization function of the HDG method. We apply this approach to HDG methods using polynomials of degree $k \geq 0$ in all of the unknowns, and show that the projection of the error in each of them superconverges with order $k+2$ when $k \geq 1$ and converges with order 1 for $k=0$. As a result, we show that the HDG methods converge with optimal order $k+1$ for all the unknowns, and that they are free from shear locking. Finally, we show that all of the numerical traces converge with order $2 k+1$. Numerical experiments validating these results are shown.
\end{abstract}

\section{INTRODUCTION}

In this paper, we provide an a priori error analysis of the hybridizable discontinuous Galerkin (HDG) methods for Timoshenko beams recently introduced by the authors in [4. The dimensionless form of the Timoshenko beam model 8 , is given by the differential equations, see ([5]),

$$
w^{\prime}=\theta-d^{2} \frac{T}{G A}, \quad \theta^{\prime}=\frac{M}{E I}, \quad M^{\prime}=T, \quad T^{\prime}=q,
$$

in $\Omega:=(0,1)$, and the boundary conditions

$$
w=w_{D}, \quad \theta=\theta_{N} \quad \text { on } \partial \Omega=\{0,1\} .
$$

Here, the unknowns are the transverse displacement $w$, the rotation of the transverse cross-section of the beam $\theta$, the bending moment $M$, and the shear force $T$. The material and geometrical properties of the beam are characterized by the shear

Received by the editor April 11, 2010 and, in revised form, October 12, 2010.

2010 Mathematics Subject Classification. Primary 65M60, 65N30, 35J30.

Key words and phrases. Discontinuous Galerkin methods, Timoshenko beams, hybridization.

The second author was partially supported by the National Science Foundation (Grant DMS0712955) and by the Minnesota Supercomputing Institute. Part of this work was done when this author was visiting the Research Institute for Mathematical Sciences, Kyoto University, Japan, during the Fall of 2009. 
modulus $G$, the cross-section area $A$, the Young modulus $E$, and the moment of inertia $I$. The transverse load is denoted by $q$. It was shown in [5] that we can assume without loss of generality that $E I$ and $G A$ are very smooth functions. The parameter $0<d<1$ represents the thickness of the beam.

The first discontinuous Galerkin (DG) methods for Timoshenko beams were introduced in [3]. The convergence properties of these methods were numerically explored in [2]. Later, in [5], these DG methods were proven to provide approximations for the displacement, rotation, bending moment and shear force simultaneously converging with the optimal order of $k+1$ when polynomials of degree $k \geq 0$ were used to define the method. For the corresponding numerical traces at the nodes, the order of convergence of $2 k+1$ was also established for $k \geq 0$. Finally, it was proved that these properties hold uniformly with respect to the thickness of the beam.

Recently, a new class of DG methods for Timoshenko beams was proposed. Indeed, motivated by the introduction of the HDG methods [6] for diffusion problems, the authors introduced, and numerically studied, the first HDG methods for Timoshenko beams [4. The main feature of the HDG methods is that their only globally coupled degrees of freedom are those of the numerical traces of some of its variables; in this case, those of the displacement $w$ and the bending moment $M$. The matrix to numerically invert is thus a highly structured square matrix of order $2 N$, where $N$ is the number of intervals in which $\Omega$ is partitioned. The size of the matrix is independent of the polynomial degree $k$ of the approximation; see 4 .

In this paper, we analyze the HDG methods proposed in 4 by using the approach proposed in [7] to study HDG methods for diffusion problems when all the variables are approximated by piecewise polynomials of degree $k \geq 0$. We show that results similar to those proven in [5] for the DG methods 3 hold for a wide class of HDG methods. The approach has three main steps. The first is to find a suitably defined projection such that the equations of the projection of the approximation errors becomes extremely simple. This is achieved by tailoring the definition of the projection to the very structure of the numerical traces of the HDG methods. The second step is to study the approximation properties of the projection in a single typical element. It is in this step that the information of the particular definition of the numerical traces is captured and allows us to determine for what choices we obtain the optimal order of convergence of $k+1$ for each of the four variables for $k \geq 0$. The third step consists of bounding the projection of the errors in terms of the approximation properties of the projection only. This step is rendered particularly concise because of the simple form of the equations of the projection of the errors.

The analysis we present here has two striking features. The first is that no positivity of the stabilization function is required; let us elaborate. In the analysis of numerical methods for symmetric elliptic problems, it is standard to take advantage of the fact that they have an underlying energy. The delicate devising of the numerical traces for the DG methods is then geared towards making sure that the discrete energy associated with the jumps of the approximation is actually positive. This is the approach taken in 5 to analyze the first DG methods for Timoshenko beams; it could have been easily taken to carry out the analysis of the methods under consideration. However, the alternative approach we present here has an unexpected and surprising feature. It is the fact that, unlike the analysis of any 
other DG method, we do not rely on the stabilization function having any positivity property.

The second striking feature is that the projection of the errors of each of all the components of the approximation superconverge with order $k+2$ when $k \geq 1$ and with order 1 for $k=0$.

The two properties above are due to the fact that we are only using duality arguments and that the problem is one-dimensional. The price to pay, however, is that the maximum meshsize of the partition, $h$, needs to be small enough.

The rest of the paper is organized as follows. In Section 2, we display the HDG methods, define the projection employed in the error analysis and state briefly our main results. Detailed proofs of these results are presented in Sections 3 (approximation properties of the projection), 4 (estimates of the projection of the errors) and 5 (estimates of the errors of the numerical traces). Numerical results verifying the theoretical orders of convergence are presented in Section [6. We end in Section 7 with some concluding remarks.

\section{Main Results}

In this section, after describing the HDG method under consideration, we state our main results regarding the convergence properties of the method.

2.1. The HDG methods. Let us describe the HDG methods under consideration; we follow 4 . We begin by introducing the notation. To each partition of the domain $\Omega$,

$$
\Omega_{h}:=\left\{\left(x_{j-1}, x_{j}\right): 0=x_{0}<x_{1}<\cdots<x_{N-1}<x_{N}=1\right\},
$$

we associate the set of nodes, $\mathscr{E}_{h}:=\left\{x_{0}, x_{1}, \ldots, x_{N}\right\}$, and the set of interior nodes, $\mathscr{E}_{h}^{\circ}:=\mathscr{E}_{h} \backslash \partial \Omega$; we also set $\partial \Omega_{h}:=\left\{\partial K: K \in \Omega_{h}\right\}$. For each element $K \in \Omega_{h}$, let $h_{K}$ denote the length of $K$, and set $h:=\max _{K \in \Omega_{h}}\left\{h_{K}\right\}$. Finally, for any given polynomial degree $k \geq 0$ and an element $K \in \Omega_{h}$, we define $\mathcal{P}^{k}(K)$ as the set of polynomials of degree less than or equal to $k$ on $K$. The space of piecewise polynomials of degree $k$ on $\Omega$ is defined accordingly as

$$
V_{h}^{k}:=\left\{v: \Omega_{h} \mapsto \mathbb{R}:\left.\quad v\right|_{K} \in \mathcal{P}^{k}(K) \text { for all } K \in \Omega_{h}\right\} .
$$

We also set

$$
L_{0}^{2}\left(\mathscr{E}_{h}\right):=\left\{\mathrm{w} \in L^{2}\left(\mathscr{E}_{h}\right): \mathrm{w}=0 \text { on } \partial \Omega\right\} .
$$

The HDG method seeks an approximation $\left(T_{h}, M_{h}, \theta_{h}, w_{h}, \widehat{M}_{h}, \widehat{w}_{h}\right)$ to the exact solution $\left(T, M, \theta, w,\left.M\right|_{\mathscr{E}_{h}},\left.w\right|_{\mathscr{E}_{h}}\right)$, in the finite dimensional space $\left[V_{h}^{k}\right]^{4} \times L^{2}\left(\mathscr{E}_{h}\right) \times$ $L^{2}\left(\mathscr{E}_{h}\right)$. It is determined by requiring that

$$
\begin{aligned}
-\left(w_{h}, v_{1}^{\prime}\right)_{\Omega_{h}}+\left\langle\widehat{w}_{h}, v_{1} n\right\rangle_{\partial \Omega_{h}} & =\left(\theta_{h}, v_{1}\right)_{\Omega_{h}}-d^{2}\left(T_{h} / G A, v_{1}\right)_{\Omega_{h}}, \\
-\left(\theta_{h}, v_{2}^{\prime}\right)_{\Omega_{h}}+\left\langle\widehat{\theta}_{h}, v_{2} n\right\rangle_{\partial \Omega_{h}} & =\left(M_{h} / E I, v_{2}\right)_{\Omega_{h}}, \\
-\left(M_{h}, v_{3}^{\prime}\right)_{\Omega_{h}}+\left\langle\widehat{M}_{h}, v_{3} n\right\rangle_{\partial \Omega_{h}} & =\left(T_{h}, v_{3}\right)_{\Omega_{h}}, \\
-\left(T_{h}, v_{4}^{\prime}\right)_{\Omega_{h}}+\left\langle\widehat{T}_{h}, v_{4} n\right\rangle_{\partial \Omega_{h}} & =\left(q, v_{4}\right)_{\Omega_{h}}, \\
\left\langle\widehat{\theta}_{h}, \mathrm{~m} n\right\rangle_{\partial \Omega_{h}} & =\left\langle\theta_{N}, \mathrm{~m} n\right\rangle_{\partial \Omega}, \\
\left\langle\widehat{T}_{h}, \mathrm{w} n\right\rangle_{\partial \Omega_{h}} & =0
\end{aligned}
$$


hold for all $\left(v_{1}, v_{2}, v_{3}, v_{4}, \mathrm{~m}, \mathrm{w}\right) \in\left[V_{h}^{k}\right]^{4} \times L^{2}\left(\mathscr{E}_{h}\right) \times L_{0}^{2}\left(\mathscr{E}_{h}\right)$. Here, the outward unit normal vectors are $n\left(x^{\mp}\right):= \pm 1$ for $x \in \mathscr{E}_{h}$. The "volume" inner product is defined as

$$
(u, v)_{\Omega_{h}}:=\sum_{j=1}^{N}(u, v)_{I_{j}} \quad \text { where } \quad(u, v)_{I_{j}}:=\int_{I_{j}} u(x) v(x) d x,
$$

and the boundary inner product is defined as

$$
\langle u, v n\rangle_{\partial \Omega_{h}}:=\sum_{j=1}^{N}\langle u, v n\rangle_{\partial I_{j}} \quad \text { where } \quad\langle u, v\rangle_{\partial I_{j}}:=u\left(x_{j}^{-}\right) v\left(x_{j}^{-}\right)+u\left(x_{j-1}^{+}\right) v\left(x_{j-1}^{+}\right) .
$$

Here, we are using the following notation. For any $\varphi \in H^{1}\left(\Omega_{h}\right)$ we set $\varphi\left(x_{\ell}^{\mp}\right):=$ $\lim _{\epsilon \downarrow 0} \varphi\left(x_{\ell} \mp \epsilon\right)$ for $x_{\ell} \in \mathscr{E}_{h}$. For any $\varphi \in L^{2}\left(\partial \Omega_{h}\right)$, the value of $\varphi$ at $x_{\ell}$ is denoted by $\varphi\left(x_{\ell}^{-}\right)$, respectively, $\varphi\left(x_{\ell}^{+}\right)$, when $x_{\ell}$ is a boundary of $I_{\ell}$, respectively, of $I_{\ell+1}$.

Note that the boundary condition (1.1b) on $\theta$ is imposed by equation (2.1e). The boundary condition (1.1b) on $w$ is imposed as follows:

$$
\widehat{w}_{h}=w_{D} \quad \text { on } \partial \Omega \text {. }
$$

To complete the definition of the HDG method, we need to express the numerical traces $\widehat{T}_{h}$ and $\widehat{\theta}_{h}$ in terms of the unknowns:

$$
\left[\begin{array}{c}
\widehat{\theta}_{h} \\
\widehat{T}_{h}
\end{array}\right]=\left[\begin{array}{c}
\theta_{h} \\
T_{h}
\end{array}\right]-\mathrm{S}\left[\begin{array}{c}
M_{h}-\widehat{M}_{h} \\
w_{h}-\widehat{w}_{h}
\end{array}\right] n,
$$

where the so-called stabilization function $\mathrm{S}$ is a matrix-valued function defined on $\partial \Omega_{h}$. It has to be suitably defined to guarantee the existence and uniqueness of the approximate solution; for details see [4.

Let us note that the hallmark of these methods lies in the fact that the only globally coupled degrees of freedom are the values of $\widehat{M}_{h}$ and $\widehat{w}_{h}$ on $\mathscr{E}_{h}$. The remaining degrees of freedom can then be recovered in an element-by-element fashion; see [4].

2.2. The projection. Next, we introduce the main tool of our error analysis, namely, a new projection,

$$
\boldsymbol{\Pi}=\left(\Pi_{T}, \Pi_{M}, \Pi_{\theta}, \Pi_{\mathfrak{W}}\right):\left[H^{1}\left(\Omega_{h}\right)\right]^{4} \rightarrow\left[V_{h}^{k}\right]^{4},
$$

associated with the HDG methods. It is a generalization of the projection introduced in [7] for the error analysis of HDG methods for second order elliptic problems.

It is defined as follows. Given a function $\left(u_{1}, u_{2}, u_{3}, u_{4}\right) \in\left[H^{1}\left(\Omega_{h}\right)\right]^{4}$ and an arbitrary subinterval $K \in \Omega_{h}$, the restriction of $\left(\Pi_{T} u_{1}, \Pi_{M} u_{2}, \Pi_{\theta} u_{3}, \Pi_{\mathfrak{W}} u_{4}\right)$ to $K$ is defined as the element of $\left[\mathcal{P}^{k}(K)\right]^{4}$ that satisfies

$$
\begin{aligned}
\left(\Pi_{T} u_{1}, v_{1}\right)_{K} & =\left(u_{1}, v_{1}\right)_{K}, \\
\left(\Pi_{M} u_{2}, v_{2}\right)_{K} & =\left(u_{2}, v_{2}\right)_{K}, \\
\left(\Pi_{\theta} u_{3}, v_{3}\right)_{K} & =\left(u_{3}, v_{3}\right)_{K}, \\
\left(\Pi_{\mathcal{W}} u_{4}, v_{4}\right)_{K} & =\left(u_{4}, v_{4}\right)_{K},
\end{aligned}
$$

for all $\left(v_{1}, v_{2}, v_{3}, v_{4}\right) \in\left[\mathcal{P}^{k-1}(K)\right]^{4}$, and

$$
\left[\begin{array}{l}
u_{3} \\
u_{1}
\end{array}\right]=\left[\begin{array}{l}
\Pi_{\theta} u_{3} \\
\Pi_{T} u_{1}
\end{array}\right]-\mathrm{S}\left[\begin{array}{c}
\Pi_{M} u_{2}-u_{2} \\
\Pi_{w} u_{4}-u_{4}
\end{array}\right] n \quad \text { on } \partial K .
$$


Note that when $k=0$, the projection is defined solely by (2.3e). Note also that the last set of equations reflects the form of the equations (2.2) defining the numerical traces $\widehat{\theta}_{h}$ and $\widehat{T}_{h}$. As we are going to see in the next subsection, this is what allows us to obtain a very simple set of equations for the projection of the errors.

Finally, let us point out that the projection is well defined under mild conditions on the stabilization function S. To see this, note that the total number of unknowns involved in the linear system that is needed to be solved for computing $\left(\Pi_{T} u_{1}, \Pi_{M} u_{2}, \Pi_{\theta} u_{3}, \Pi_{\mathfrak{w}} u_{4}\right)$ is $4(k+1)$ since each component of the projection has $k+1$ degrees of freedom. On the other hand, the total number of linearly independent equations provided by the definition of the projection is also $4(k+1)$. The existence and uniqueness of the projection then follows from the approximation properties of the projection; see below.

2.3. The equations for the projection of the errors. As we said in the Introduction, the projection should be devised in such a way that the equations of the projection of the errors be as simple as possible. Let us show that this is indeed the case.

So, since the exact solution (T, $M, \theta, w)$ of the governing equations (1.1a) satisfies the formulation of the HDG approximation, (2.1), we immediately see that the equations for the errors

$$
e_{u}:=u-u_{h} \quad \text { and } \quad \widehat{e}_{u}:=u-\widehat{u}_{h} \quad \text { for } u=T, M, \theta, w
$$

are

$$
\begin{aligned}
-\left(e_{\mathcal{W}}, v_{1}^{\prime}\right)_{\Omega_{h}}+\left\langle\widehat{e}_{\mathcal{W}}, v_{1} n\right\rangle_{\partial \Omega_{h}} & =\left(e_{\theta}, v_{1}\right)_{\Omega_{h}}-d^{2}\left(e_{T} / G A, v_{1}\right)_{\Omega_{h}}, \\
-\left(e_{\theta}, v_{2}^{\prime}\right)_{\Omega_{h}}+\left\langle\widehat{e}_{\theta}, v_{2} n\right\rangle_{\partial \Omega_{h}} & =\left(e_{M} / E I, v_{2}\right)_{\Omega_{h}}, \\
-\left(e_{M}, v_{3}^{\prime}\right)_{\Omega_{h}}+\left\langle\widehat{e}_{M}, v_{3} n\right\rangle_{\partial \Omega_{h}} & =\left(e_{T}, v_{3}\right)_{\Omega_{h}}, \\
-\left(e_{T}, v_{4}^{\prime}\right)_{\Omega_{h}}+ & \left\langle\widehat{e}_{T}, v_{4} n\right\rangle_{\partial \Omega_{h}}=0 \\
\left\langle\widehat{e}_{\theta}, \mathrm{m} n\right\rangle_{\partial \Omega_{h}} & =0 \\
\left\langle\widehat{e}_{T}, \mathrm{w} n\right\rangle_{\partial \Omega_{h}} & =0,
\end{aligned}
$$

hold for all $\left(v_{1}, v_{2}, v_{3}, v_{4}, \mathrm{~m}, \mathrm{w}\right) \in\left[V_{h}^{k}\right]^{4} \times L^{2}\left(\mathscr{E}_{h}\right) \times L_{0}^{2}\left(\mathscr{E}_{h}\right)$. Hence, setting

$$
\delta_{u}:=u-\Pi_{u} u \quad \text { for } \quad u=T, M, \theta, w,
$$

we obtain

$$
\begin{aligned}
(2.4 \mathrm{a})-\left(\Pi_{\mathcal{W}} e_{\mathcal{W}}, v_{1}^{\prime}\right)_{\Omega_{h}}+\left\langle\widehat{e}_{\mathcal{W}}, v_{1} n\right\rangle_{\partial \Omega_{h}}= & \left(\Pi_{\theta} e_{\theta}, v_{1}\right)_{\Omega_{h}}-d^{2}\left(\Pi_{T} e_{T} / G A, v_{1}\right)_{\Omega_{h}} \\
& +\left(\delta_{\theta}, v_{1}\right)_{\Omega_{h}}-d^{2}\left(\delta_{T} / G A, v_{1}\right)_{\Omega_{h}}, \\
(2.4 \mathrm{~b})-\left(\Pi_{\theta} e_{\theta}, v_{2}^{\prime}\right)_{\Omega_{h}}+\left\langle\widehat{e}_{\theta}, v_{2} n\right\rangle_{\partial \Omega_{h}}= & \left(\Pi_{M} e_{M} / E I, v_{2}\right)_{\Omega_{h}}+\left(\delta_{M} / E I, v_{2}\right)_{\Omega_{h}}, \\
(2.4 \mathrm{c})-\left(\Pi_{M} e_{M}, v_{3}^{\prime}\right)_{\Omega_{h}}+\left\langle\widehat{e}_{M}, v_{3} n\right\rangle_{\partial \Omega_{h}}= & \left(\Pi_{T} e_{T}, v_{3}\right)_{\Omega_{h}}+\left(\delta_{T}, v_{3}\right)_{\Omega_{h}}, \\
(2.4 \mathrm{~d})-\left(\Pi_{T} e_{T}, v_{4}^{\prime}\right)_{\Omega_{h}}+\left\langle\widehat{e}_{T}, v_{4} n\right\rangle_{\partial \Omega_{h}}=0, & 0, \\
(2.4 \mathrm{e}) \quad\left\langle\widehat{e}_{\theta}, \mathrm{m} n\right\rangle_{\partial \Omega_{h}}=0, & \left.\widehat{e}_{T}, \mathrm{w} n\right\rangle_{\partial \Omega_{h}}=0,
\end{aligned}
$$

for all $\left(v_{1}, v_{2}, v_{3}, v_{4}, \mathrm{~m}, \mathrm{w}\right) \in\left[V_{h}^{k}\right]^{4} \times L^{2}\left(\mathscr{E}_{h}\right) \times L_{0}^{2}\left(\mathscr{E}_{h}\right)$. Note that we have used the orthogonality property of the projection (2.3) in each of the first terms of the first four equations.

To complete the error equations, we have to add the boundary conditions

$$
\widehat{e}_{W}=0 \text { on } \partial \Omega \text {, }
$$


as well as the equations relating the errors inside the elements to the errors of the numerical traces, namely,

$$
\left[\begin{array}{c}
\widehat{e}_{\theta} \\
\widehat{e}_{T}
\end{array}\right]=\left[\begin{array}{c}
\Pi_{\theta} e_{\theta} \\
\Pi_{\theta} e_{T}
\end{array}\right]-\mathrm{S}\left[\begin{array}{c}
\Pi_{M} e_{M}-\widehat{e}_{M} \\
\Pi_{\mathcal{w}} e_{\mathfrak{w}}-\widehat{e}_{\mathfrak{w}}
\end{array}\right] n \quad \text { on } \partial \Omega_{h} .
$$

These equations hold as a direct consequence of the parallelism between the definition of the numerical traces of the HDG method, 2.2b), and the definition of the projection, 2.3e).

The simplicity of the above equations we have been referring to resides in the fact that they differ from the HDG approximation only in the definition of their righthand sides which, moreover, involve only volume integrals of the approximation errors $\delta_{T}, \delta_{M}$ and $\delta_{\theta}$.

2.4. Approximation properties of the projection $\Pi$. In this subsection we state a theorem displaying the approximation properties of the projection $\Pi=$ $\left(\Pi_{T}, \Pi_{M}, \Pi_{\theta}, \Pi_{\mathcal{W}}\right)$. First, we need to introduce some notation. Let $K=\left(x_{L}, x_{R}\right)$ be an element of $\Omega_{h}$. For any function $z$ on $K$, we define $z^{-}:=z\left(x_{L}\right), z^{+}:=z\left(x_{R}\right)$, We denote the usual norm and seminorm on a Sobolev space $H^{s}(D)$ by $\|\cdot\|_{s, D}$ and $|\cdot|_{s, D}$, respectively. We drop the first subindex if $s=0$, and the second one if $D=\Omega$ or $D=\Omega_{h}$. We also define the seminorm of a vector-valued function $\left(u_{1}, u_{2}, u_{3}, u_{4}\right)$ as

$$
\left|\left(u_{1}, u_{2}, u_{3}, u_{4}\right)\right|_{s, D}:=\left|u_{1}\right|_{s, D}+\left|u_{2}\right|_{s, D}+\left|u_{3}\right|_{s, D}+\left|u_{4}\right|_{s, D} .
$$

Its norm is defined similarly.

Theorem 2.1. On each $K \in \Omega_{h}$ with stabilization function $\mathrm{S}$, we have for any $s$ in $[1, k+1]$ that

$$
\left\|\left(\delta_{T}, \delta_{M}, \delta_{\theta}, \delta_{w}\right)\right\| \leq C C_{\mathrm{S}} h^{s}|(T, M, \theta, w)|_{s} .
$$

Here, $C$ is a constant independent of the discretization parameters and $(T, M, \theta, w)$, and $C_{\mathrm{S}}$ is given by

$$
\begin{aligned}
C_{\mathrm{S}} & :=\left\|\left(\mathrm{S}^{+}+\mathrm{S}^{-}\right)^{-1}\right\|_{\infty}+\left\|\left(\mathrm{S}^{+}+\mathrm{S}^{-}\right)^{-1} \mathrm{~S}^{+}\right\|_{\infty}+\left\|\left(\mathrm{S}^{+} \mathrm{S}^{-}\right)^{-1} \mathrm{~S}^{-}\right\|_{\infty} \\
& +\left\|\mathrm{S}^{+}\left(\mathrm{S}^{+}+\mathrm{S}^{-}\right)^{-1}\right\|_{\infty}+\left\|\mathrm{S}^{-}\left(\mathrm{S}^{+}+\mathrm{S}^{-}\right)^{-1}\right\|_{\infty}+\left\|\mathrm{S}^{+}\left(\mathrm{S}^{+}+\mathrm{S}^{-}\right)^{-1} \mathrm{~S}^{-}\right\|_{\infty} .
\end{aligned}
$$

A detailed proof of this result is given in Section 3 . Let us note that we stated the above result for $(T, M, \theta, w)$ merely for notational convenience. In fact, the result remains valid if we replace $(T, M, \theta, w)$ with any $\left(u_{1}, u_{2}, u_{3}, u_{4}\right) \in\left[H^{s+1}(K)\right]^{4}$.

2.5. Superconvergence of the projection of the errors. Here, we present an estimate of the following norm of the projection of the errors,

$$
\|\Pi e\|:=\left(\left\|\Pi_{T} e_{T}\right\|^{2}+\left\|\Pi_{M} e_{M}\right\|^{2}+\left\|\Pi_{\theta} e_{\theta}\right\|^{2}+\left\|\Pi_{\mathcal{W}} e_{\mathcal{W}}\right\|^{2}\right)^{\frac{1}{2}},
$$

in terms of the following norm of the approximation error of the projection

$$
\|\boldsymbol{\delta}\|:=\left(\left\|\delta_{T}\right\|^{2}+\left\|\delta_{M}\right\|^{2}+\left\|\delta_{\theta}\right\|^{2}+\left\|\delta_{\mathfrak{w}}\right\|^{2}\right)^{\frac{1}{2}} .
$$

It is stated in terms of the solution of the so-called dual problem we define next. 
For any given $\left(\eta_{T}, \eta_{M}, \eta_{\theta}, \eta_{\mathcal{W}}\right) \in\left[L^{2}(\Omega)\right]^{4}$, the associated dual-problem is:

$$
\begin{aligned}
\psi_{\mathfrak{w}}^{\prime} & =\psi_{\theta}-d^{2} \psi_{T} / G A+\eta_{T} & & \text { in } \Omega, \\
\psi_{\theta}^{\prime} & =\psi_{M} / E I-\eta_{M} & & \text { in } \Omega, \\
\psi_{M}^{\prime} & =\psi_{T}+\eta_{\theta} & & \text { in } \Omega, \\
\psi_{T}^{\prime} & =-\eta_{\mathfrak{w}} & & \text { in } \Omega, \\
\psi_{\mathfrak{w}} & =0 & & \text { on } \partial \Omega, \\
\psi_{\theta} & =0 & & \text { on } \partial \Omega .
\end{aligned}
$$

The key inequality we use about the solution of this problem is the following elliptic regularity result:

$$
\left\|\psi_{T}\right\|_{1}+\left\|\psi_{M}\right\|_{1}+\left\|\psi_{\theta}\right\|_{1}+\left\|\psi_{\mathcal{W}}\right\|_{1} \leq C_{\mathrm{reg}}\left\|\left(\eta_{T}, \eta_{M}, \eta_{\theta}, \eta_{\mathfrak{W}}\right)\right\|,
$$

where the constant $C_{\mathrm{reg}}$ is independent of the data $\left(\eta_{T}, \eta_{M}, \eta_{\theta}, \eta_{\mathcal{W}}\right)$ and of the thickness $d$. A detailed proof of this lemma can be found in Appendix A

We are now ready to state our main result.

Theorem 2.2. For $k \geq 1$, we have that, if $h$ is sufficiently small, then

$$
\|\boldsymbol{\Pi} e\| \leq C C_{\mathrm{reg}} h\|\boldsymbol{\delta}\| \text {. }
$$

For $k=0$, we have

$$
\|\Pi \boldsymbol{\Pi}\| \leq C C_{\text {reg }}\|\boldsymbol{\delta}\| .
$$

Here $C$ is a constant independent of the data of the problem and of the discretization parameters.

2.6. A priori error estimates. Next we present an estimate for the following norm of the errors:

$$
\|\boldsymbol{e}\|:=\left(\left\|e_{T}\right\|^{2}+\left\|e_{M}\right\|^{2}+\left\|e_{\theta}\right\|^{2}+\left\|e_{\mathcal{W}}\right\|^{2}\right)^{\frac{1}{2}},
$$

which is a direct consequence of the last result.

Theorem 2.3. Suppose that the exact solution $(T, M, \theta, w)$ of (1.1a) belongs to $\left[H^{k+1}\left(\Omega_{h}\right)\right]^{4}$. Then, for $k \geq 1$ and $h$ sufficiently small, we have

$$
\|\boldsymbol{e}\| \leq\left(1+C C_{\mathrm{reg}} h\right)\|\boldsymbol{\delta}\| .
$$

For $k=0$, we have

$$
\|\boldsymbol{e}\| \leq\left(1+C C_{\mathrm{reg}}\right)\|\boldsymbol{\delta}\| .
$$

Here $C$ is a constant independent of the data of the problem and of the discretization parameters.

Note that the error estimate appearing in the above theorem shows that, if the matrix-valued function $\mathrm{S}$ is chosen is such a way that $C_{\mathrm{S}}$ is uniformly bounded, the HDG method is optimally convergent, that is, $\left\|u-u_{h}\right\|=\mathcal{O}\left(h^{k+1}\right)$ for smooth solutions for each $u=T, M, \theta, w$, and locking-free. The method is locking-free because the constant $C_{\mathrm{S}}$ does not depend on the parameter $d$ and because the seminorms appearing on the right-hand side of the estimate can be bounded uniformly with respect to $d$; see $[5$. 
2.7. Superconvergence at the nodes. Our next result states that the numerical traces of the HDG solution superconverge. To state this result we need to introduce the Green's functions associated with the problem under consideration. For any superindex $\star=T, M, \theta, \mathfrak{w}$ and any $y \in(0,1)$ we define $\left(\Phi_{T, y}^{\star}, \Phi_{M, y}^{\star}, \Phi_{\theta, y}^{\star}, \Phi_{\mathcal{W}, y}^{\star}\right)$ as the solution of

$$
\begin{array}{r}
\frac{d \Phi_{\mathcal{W}, y}^{\star}}{d x}=\Phi_{\theta, y}^{\star}-d^{2} \frac{\Phi_{T, y}^{\star}}{G A}, \quad \frac{d \Phi_{\theta, y}^{\star}}{d x}=\frac{\Phi_{M, y}^{\star}}{E I}, \\
\frac{d \Phi_{M, y}^{\star}}{d x}=\Phi_{T, y}^{\star}, \quad \frac{d \Phi_{T, y}^{\star}}{d x}=0,
\end{array}
$$

in $(0, y) \cup(y, 1)$ that satisfies the boundary conditions

$$
\Phi_{\mathcal{W}, y}^{\star}(0)=\Phi_{\mathcal{W}, y}^{\star}(1)=\Phi_{\theta, y}^{\star}(0)=\Phi_{\theta, y}^{\star}(1)=0,
$$

and the jump conditions

$$
\begin{aligned}
& \llbracket \Phi_{\mathcal{W}, y}^{\star} \rrbracket(y)=-\delta_{\star T}, \quad \llbracket \Phi_{\theta, y}^{\star} \rrbracket(y)=\delta_{\star M}, \\
& \llbracket \Phi_{M, y}^{\star} \rrbracket(y)=-\delta_{\star \theta}, \quad \llbracket \Phi_{T, y}^{\star} \rrbracket(y)=\delta_{\star \mathcal{W}} .
\end{aligned}
$$

Here, $\delta_{a b}=1$ if $a=b$, and $\delta_{a b}=0$ otherwise. The jump operator, $\llbracket \cdot \rrbracket$, is defined by

$$
\llbracket \varphi \rrbracket(x):=\varphi\left(x^{-}\right)-\varphi\left(x^{+}\right) \quad \text { for } x \in \mathscr{E}_{h}
$$

where $\varphi\left(x^{\mp}\right):=\lim _{\epsilon \downarrow 0} \varphi(x \mp \epsilon)$. We define, for $z \in\{0,1\}$,

$$
\left(\Phi_{T, z}^{\star}, \Phi_{M, z}^{\star}, \Phi_{\theta, z}^{\star}, \Phi_{\mathcal{W}, z}^{\star}\right):=\lim _{y \rightarrow z}\left(\Phi_{T, y}^{\star}, \Phi_{M, y}^{\star}, \Phi_{\theta, y}^{\star}, \Phi_{\mathcal{W}, y}^{\star}\right) .
$$

We are now ready to present our superconvergence result of the numerical traces.

Theorem 2.4. Under the same assumptions as in Theorem 2.3, we have

$$
\left|\left(u-\widehat{u}_{h}\right)\left(x_{i}\right)\right| \leq C_{k-1} h^{k}|(T, M, \theta, w)|_{k+1}\left\|\boldsymbol{\delta}_{i}^{u}\right\|+C\|\boldsymbol{e}\|\left\|\boldsymbol{\delta}_{i}^{u}\right\|
$$

for $u=T, M, \theta, w$, and $i=0,1, \ldots, N$. Here

$$
\begin{aligned}
\delta_{i}^{u}:= & \left(\left\|\Phi_{T, x_{i}}^{u}-\Pi_{T} \Phi_{T, x_{i}}^{u}\right\|^{2}+\left\|\Phi_{M, x_{i}}^{u}- \pm \Phi_{M, x_{i}}^{u}\right\|^{2}\right. \\
& \left.+\left\|\Phi_{\theta, x_{i}}^{u}-\Pi_{\theta} \Phi_{\theta, x_{i}}^{u}\right\|^{2}+\left\|\Phi_{\mathcal{W}, x_{i}}^{u}-\Pi_{\mathcal{W}} \Phi_{\mathcal{W}, x_{i}}^{u}\right\|^{2}\right)^{\frac{1}{2}},
\end{aligned}
$$

and $C_{k-1}$ is a constant that depends solely on the polynomial degree $k$.

Note that, for any given $k \geq 0$, if $q, E I, G A$ are very smooth functions in $\Omega_{h}$, the exact solution $(T, M, \theta, w)$ belongs to $\left[H^{k+1}\left(\Omega_{h}\right)\right]^{4}$; see [5. This regularity result is also valid for the Green's functions since in this case we take $q=0$. Hence, we can assume that $\left(\Phi_{T, x_{i}}^{u}, \Phi_{M, x_{i}}^{u}, \Phi_{\theta, x_{i}}^{u}, \Phi_{\mathcal{W}, x_{i}}^{u}\right)$ belongs to $\left[H^{k+1}\left(\Omega_{h}\right)\right]^{4}$. As a consequence, $\left\|\boldsymbol{\delta}_{i}^{u}\right\|=\boldsymbol{O}\left(h^{k+1}\right)$ and the above result states that, if the constant $C_{\mathrm{S}}$ is uniformly bounded, all of the numerical traces superconverge with order $2 k+1$ at each node. A similar result was proved for the DG methods for Timoshenko beams studied in 5] and [1.

Let us point out that if the data $E I$ and $G A$ are constants on each $K \in \Omega_{h}$, then, for $k \geq 3$, Theorem 2.4 implies that $\widehat{e}_{u}\left(x_{i}\right)=0$, for $u=T, M, \theta$, or $w$ and for any node $x_{i}$. Indeed, in this case the Green's functions are piecewise polynomials of degree at most 3 and hence $\left|\left(\Phi_{T, x_{i}}^{u}, \Phi_{M, x_{i}}^{u}, \Phi_{\theta, x_{i}}^{u}, \Phi_{\mathcal{W}, x_{i}}^{u}\right)\right|_{k+1}=0$.

An immediate application of the superconvergence result of Theorem 2.4 is an element-by-element postprocessing of the approximate solution provided by the HDG method. All of the four components of the postprocessed solution converge 
to the exact solution with order $2 k+1$, not only at the nodes, but also uniformly at the interior of $\Omega_{h}$. For details, see [2].

\section{Approximation properties of the projection: Proof of Theorem 2.1}

In this section we provide a detailed proof of Theorem 2.1. We only give the proof for $k \geq 1$. The proof for $k=0$ is easier.

Fix an interval $K \in \mathcal{T}_{h}$ and set $d_{u}:=u_{k}-\Pi_{u} u, g_{u}:=u-u_{k}$, for $u=T, M, \theta, w$; Here $u_{k}$ denotes the $L^{2}$-projection into $\mathcal{P}_{k}(K)$. Since $\delta_{u}=g_{u}+d_{u}$, we only need to estimate $d_{u}$. To do that, we proceed as follows. From the definition of the projection (2.3a)-(2.3e) and the definition of the $L^{2}$-projection into $\mathcal{P}_{k}(K)$, we have

$$
\left(d_{T}, v_{1}\right)_{K}=\left(d_{M}, v_{2}\right)_{K}=\left(d_{\theta}, v_{3}\right)_{K}=\left(d_{\mathcal{W}}, v_{4}\right)_{K}=0,
$$

for all $\left(v_{1}, v_{2}, v_{3}, v_{4}\right) \in\left[\mathcal{P}^{k-1}(K)\right]^{4}$, and

$$
\left[\begin{array}{l}
d_{\theta} \\
d_{T}
\end{array}\right] n-\mathrm{S}\left[\begin{array}{l}
d_{M} \\
d_{\mathcal{W}}
\end{array}\right]=\left[\begin{array}{l}
g_{\theta} \\
g_{T}
\end{array}\right] n-\mathrm{S}\left[\begin{array}{l}
g_{M} \\
g_{\mathcal{W}}
\end{array}\right] \quad \text { on } \partial K .
$$

By equations (3.1a), we see that we can write $d_{u}=C_{u} L_{k}$ for $u=T, M, \theta$, w, where $L_{k}$ denotes the scaled Legendre polynomial of degree $k$. Hence, we can write (3.1b) in the following form,

$$
\left[\begin{array}{cc}
\mathrm{I} & -\mathrm{S}^{+} \\
\mathrm{I} & \mathrm{S}^{-}
\end{array}\right]\left[\begin{array}{c}
C_{\theta} \\
C_{T} \\
C_{M} \\
C_{\mathcal{W}}
\end{array}\right]=\left[\begin{array}{c}
{\left[\begin{array}{c}
g_{\theta} \\
g_{T}
\end{array}\right]^{+}-\mathrm{S}^{+}\left[\begin{array}{c}
g_{M} \\
g_{\mathfrak{W}}
\end{array}\right]^{+}} \\
(-1)^{k}\left[\begin{array}{c}
g_{\theta} \\
g_{T}
\end{array}\right]^{-}+(-1)^{k} \mathrm{~S}^{-}\left[\begin{array}{l}
g_{M} \\
g_{\mathfrak{W}}
\end{array}\right]^{-}
\end{array}\right],
$$

from the above equation we can see that the system has a unique solution if and only if the matrix $\left(\mathrm{S}^{+}+\mathrm{S}^{-}\right)$is not singular. Hence we obtain, after some algebraic manipulation,

$$
\begin{aligned}
{\left[\begin{array}{l}
C_{M} \\
C_{\mathfrak{w}}
\end{array}\right]=} & (-1)^{k}\left(\mathrm{~S}^{-}+\mathrm{S}^{+}\right)^{-1}\left[\begin{array}{c}
g_{\theta} \\
g_{T}
\end{array}\right]^{-}+(-1)^{k}\left(\mathrm{~S}^{-}+\mathrm{S}^{+}\right)^{-1} \mathrm{~S}^{-}\left[\begin{array}{c}
g_{M} \\
g_{\mathfrak{w}}
\end{array}\right]^{-} \\
& -\left(\mathrm{S}^{-}+\mathrm{S}^{+}\right)^{-1}\left[\begin{array}{c}
g_{\theta} \\
g_{T}
\end{array}\right]^{+}+\left(\mathrm{S}^{-}+\mathrm{S}^{+}\right)^{-1} \mathrm{~S}^{+}\left[\begin{array}{c}
g_{M} \\
g_{\mathfrak{w}}
\end{array}\right]^{+}, \\
{\left[\begin{array}{c}
C_{\theta} \\
C_{T}
\end{array}\right]=} & (-1)^{k} \mathrm{~S}^{+}\left(\mathrm{S}^{-}+\mathrm{S}^{+}\right)^{-1}\left[\begin{array}{c}
g_{\theta} \\
g_{T}
\end{array}\right]^{-}+(-1)^{k} \mathrm{~S}^{+}\left(\mathrm{S}^{-}+\mathrm{S}^{+}\right)^{-1} \mathrm{~S}^{-}\left[\begin{array}{l}
g_{M} \\
g_{\mathfrak{w}}
\end{array}\right]^{-} \\
& +\mathrm{S}^{-}\left(\mathrm{S}^{-}+\mathrm{S}^{+}\right)^{-1}\left[\begin{array}{c}
g_{\theta} \\
g_{T}
\end{array}\right]^{+}-\mathrm{S}^{+}\left(\mathrm{S}^{-}+\mathrm{S}^{+}\right)^{-1} \mathrm{~S}^{-}\left[\begin{array}{c}
g_{M} \\
g_{\mathfrak{w}}
\end{array}\right]^{+},
\end{aligned}
$$

and conclude that

$$
\begin{aligned}
\left\|\left(d_{T}, d_{M}, d_{\theta}, d_{\mathfrak{w}}\right)\right\|_{K} & =\left\|L_{k}\right\|_{K}\left(\left|C_{T}\right|+\left|C_{M}\right|+\left|C_{\theta}\right|+\left|C_{\mathfrak{w}}\right|\right) \\
& \leq C_{\mathrm{S}}\left\|L_{k}\right\|_{K}\left\|\left(g_{T}, g_{M}, g_{\theta}, g_{\mathfrak{w}}\right)\right\|_{\partial K} \\
& \leq C_{\mathrm{S}} h^{1 / 2}\left\|\left(g_{T}, g_{M}, g_{\theta}, g_{\mathfrak{w}}\right)\right\|_{\partial K} \\
& \leq C C_{\mathrm{S}} h^{s}|(T, M, \theta, w)|_{s, K},
\end{aligned}
$$

for all $1 \leq s \leq k+1$, by the trace inequality and the approximation properties of the $L^{2}$-projection.

By the triangle inequality, we have

$$
\left\|\left(\delta_{T}, \delta_{M}, \delta_{\theta}, \delta_{\mathcal{W}}\right)\right\|_{K} \leq\left\|d_{T}, d_{M}, d_{\theta}, d_{\mathfrak{w}}\right\|_{K}+\left\|g_{T}, g_{M}, g_{\theta}, g_{\mathfrak{w}}\right\|_{K},
$$


and the estimate of Theorem 2.1 readily follows. This completes the proof.

4. Estimates of the projection of the errors: Proof of Theorem 2.2

This subsection is devoted to the proof of Theorem 2.2. We proceed in two steps. In the first, we use a key identity obtained by duality to prove Theorem 2.2 In the second, we prove the identity.

4.1. Step 1: The duality identity and the proof of Theorem 2.2, Our proof is based on the following auxiliary result.

Lemma 4.1. For any $\left(\eta_{T}, \eta_{M}, \eta_{\theta}, \eta_{w}\right) \in\left[L^{2}\left(\Omega_{h}\right)\right]^{4}$, set

$$
\mathcal{E}:=\left(\Pi_{T} e_{T}, \eta_{T}\right)_{\Omega_{h}}+\left(\Pi_{M} e_{M}, \eta_{M}\right)_{\Omega_{h}}+\left(\Pi_{\theta} e_{\theta}, \eta_{\theta}\right)_{\Omega_{h}}+\left(\Pi_{\mathcal{W}} e_{\mathcal{W}}, \eta_{\mathcal{W}}\right)_{\Omega_{h}} .
$$

Then

$$
\begin{aligned}
\mathcal{E}= & \left(\delta_{T}, \Pi_{\theta} \psi_{\theta}\right)_{\Omega_{h}} & -\left(\Pi_{T} e_{T}, \delta_{\psi_{\theta}}\right)_{\Omega_{h}} \\
& -\left(\delta_{T}, d^{2} \Pi_{T} \psi_{T} / G A\right)_{\Omega_{h}} & +\left(\Pi_{T} e_{T}, d^{2} \delta_{\psi_{T}} / G A\right)_{\Omega_{h}} \\
& -\left(\delta_{M}, \Pi_{M} \psi_{M} / E I\right)_{\Omega_{h}} & +\left(\Pi_{M} e_{M}, \delta_{\psi_{M}} / E I\right)_{\Omega_{h}} \\
& +\left(\delta_{\theta}, \Pi_{T} \psi_{T}\right)_{\Omega_{h}} & -\left(\Pi_{\theta} e_{\theta}, \delta_{\psi_{T}}\right)_{\Omega_{h}} .
\end{aligned}
$$

Here, on each $K$, we take $\mathrm{S}^{t}$ as the stabilization function for defining the projection $\left(\Pi_{T} \psi_{T}, \Pi_{M} \psi_{M}, \Pi_{\theta} \psi_{\theta}, \Pi_{\mathfrak{W}} \psi_{w}\right)$.

We delay the proof of this identity to the end of this subsection. We are now ready to prove Theorem 2.2 .

Proof of Theorem 2.2. We first consider the case $k \geq 1$. Setting

$$
\left(\eta_{T}, \eta_{M}, \eta_{\theta}, \eta_{\mathcal{W}}\right)=\left(\Pi_{T} e_{T}, \Pi_{M} e_{M}, \Pi_{\theta} e_{\theta}, \Pi_{\mathcal{W}} e_{\mathcal{W}}\right)
$$

in the identity of Lemma 4.1 gives

$$
\begin{array}{rll}
\|\boldsymbol{\Pi} \boldsymbol{e}\|^{2}= & \left(\delta_{T}, \Pi_{\theta} \psi_{\theta}\right)_{\Omega_{h}} & -\left(\Pi_{T} e_{T}, \delta_{\psi_{\theta}}\right)_{\Omega_{h}} \\
& -\left(\delta_{T}, d^{2} \Pi_{T} \psi_{T} / G A\right)_{\Omega_{h}} & +\left(\Pi_{T} e_{T}, d^{2} \delta_{\psi_{T}} / G A\right)_{\Omega_{h}} \\
& -\left(\delta_{M}, \Pi_{M} \psi_{M} / E I\right)_{\Omega_{h}} & +\left(\Pi_{M} e_{M}, \delta_{\psi_{M}} / E I\right)_{\Omega_{h}} \\
& +\left(\delta_{\theta}, \Pi_{T} \psi_{T}\right)_{\Omega_{h}} & -\left(\Pi_{\theta} e_{\theta}, \delta_{\psi_{T}}\right)_{\Omega_{h}} .
\end{array}
$$

Using the fact that $\Pi_{u} \psi_{u}=\psi_{u}-\delta_{\psi_{u}}$ for $u=T, M, \theta$, and $w$, we get

$$
\|\Pi \boldsymbol{e}\|^{2}=T_{1}+T_{2}+T_{3}+T_{4}
$$

where

$$
\begin{array}{lll}
T_{1}=\left(\delta_{T}, \psi_{\theta}\right)_{\Omega_{h}} & -\left(\delta_{T}, \delta_{\psi_{\theta}}\right)_{\Omega_{h}} & -\left(\Pi_{T} e_{T}, \delta_{\psi_{\theta}}\right)_{\Omega_{h}} \\
T_{2}=-\left(\delta_{T}, d^{2} \psi_{T} / G A\right)_{\Omega_{h}} & +\left(\delta_{T}, d^{2} \delta_{\psi_{T}} / G A\right)_{\Omega_{h}} & +\left(\Pi_{T} e_{T}, d^{2} \delta_{\psi_{T}} / G A\right)_{\Omega_{h}} \\
T_{3}=-\left(\delta_{M}, \psi_{M} / E I\right)_{\Omega_{h}} & +\left(\delta_{M}, \delta_{\psi_{M}} / E I\right)_{\Omega_{h}} & +\left(\Pi_{M} e_{M}, \delta_{\psi_{M}} / E I\right)_{\Omega_{h}} \\
T_{4}=\left(\delta_{\theta}, \psi_{T}\right)_{\Omega_{h}} & -\left(\delta_{\theta}, \delta_{\psi_{T}}\right)_{\Omega_{h}} & -\left(\Pi_{\theta} e_{\theta}, \delta_{\psi_{T}}\right)_{\Omega_{h}} .
\end{array}
$$


By the orthogonality property of the projection, (2.3), we can rewrite these equation as

$$
\begin{aligned}
T_{1}=\left(\delta_{T}, \psi_{\theta}-\left(\psi_{\theta}\right)_{k-1}\right)_{\Omega_{h}} & -\left(\delta_{T}, \delta_{\psi_{\theta}}\right)_{\Omega_{h}}-\left(\Pi_{T} e_{T}, \delta_{\psi_{\theta}}\right)_{\Omega_{h}} \\
T_{2}=-\left(\delta_{T}, d^{2}\left(\psi_{T} / G A-\left(\psi_{T} / G A\right)_{k-1}\right)\right)_{\Omega_{h}} & +\left(\delta_{T}, d^{2} \delta_{\psi_{T}} / G A\right)_{\Omega_{h}} \\
& +\left(\Pi_{T} e_{T}, d^{2} \delta_{\psi_{T}} / G A\right)_{\Omega_{h}} \\
T_{3}=-\left(\delta_{M}, \psi_{M} / E I-\left(\psi_{M} / E I\right)_{k-1}\right)_{\Omega_{h}} & +\left(\delta_{M}, \delta_{\psi_{M}} / E I\right)_{\Omega_{h}} \\
& +\left(\Pi_{M} e_{M}, \delta_{\psi_{M}} / E I\right)_{\Omega_{h}} \\
T_{4}=\left(\delta_{\theta}, \psi_{T}-\left(\psi_{T}\right)_{k-1}\right)_{\Omega_{h}} & -\left(\delta_{\theta}, \delta_{\psi_{T}}\right)_{\Omega_{h}}-\left(\Pi_{\theta} e_{\theta}, \delta_{\psi_{T}}\right)_{\Omega_{h}} .
\end{aligned}
$$

An estimate on $\|\boldsymbol{\Pi} \boldsymbol{e}\|$ now follows by estimating $T_{i}$ for $i=1,2,3,4$. We only show the details of how to estimate $T_{2}$, since the remaining terms can be estimated in a similar fashion. Applying the Cauchy-Schwarz inequality to each term in $T_{2}$, we get

$$
\left|T_{2}\right| \leq\left\|\delta_{T}\right\|\left\|d^{2}\left(\psi_{T} / G A-\left(\psi_{T} / G A\right)_{k-1}\right)\right\|+\left(\left\|\delta_{T}\right\|+\left\|\Pi_{T} e_{T}\right\|\right)\left\|d^{2} \delta_{\psi_{T}} / G A\right\| .
$$

By the approximation properties of the $L^{2}$-projection, we get that

$$
\left|T_{2}\right| \leq C h d^{2}\left\|\delta_{T}\right\|\left\|\psi_{T} / G A\right\|_{1}+\left\|d^{2} / G A\right\|_{\infty}\left(\left\|\delta_{T}\right\|+\left\|\Pi_{T} e_{T}\right\|\right)\left\|\delta_{\psi_{T}}\right\|,
$$

by the fact that $0<d<1, G A$ is very smooth, and by Theorem 2.1 we get that

$$
\left|T_{2}\right| \leq C h\left\|\delta_{T}\right\|\left\|\psi_{T}\right\|_{1}\|1 / G A\|_{1}+C h\left(\left\|\delta_{T}\right\|+\left\|\Pi_{T} e_{T}\right\|\right)\left|\left(\psi_{T}, \psi_{M}, \psi_{\theta}, \psi_{\mathfrak{W}}\right)\right|_{1} .
$$

By the elliptic regularity estimate (2.7), we have $\left|\left(\psi_{T}, \psi_{M}, \psi_{\theta}, \psi_{w}\right)\right|_{1} \leq C_{\text {reg }}\|\mathbf{\Pi} \boldsymbol{e}\|$, we get that

$$
\left|T_{2}\right| \leq C C_{\mathrm{reg}} h\left\|\delta_{T}\right\|\|\Pi e\|+C C_{\mathrm{reg}} h\|\Pi e\|^{2} .
$$

The remaining terms $T_{1}, T_{3}$, and $T_{4}$ can be estimated similarly, and hence we obtain

$$
\|\boldsymbol{\Pi} \boldsymbol{e}\|^{2} \leq\left|T_{1}\right|+\left|T_{2}\right|+\left|T_{3}\right|+\left|T_{4}\right| \leq C C_{\mathrm{reg}} h\|\boldsymbol{\delta}\|\|\boldsymbol{\Pi} \boldsymbol{e}\|+C C_{\mathrm{reg}} h\|\boldsymbol{\Pi} \boldsymbol{e}\|^{2} .
$$

If we assume that $h$ is small enough so that $C C_{\text {reg }} h<1$ then

$$
\|\boldsymbol{\Pi} \boldsymbol{e}\|^{2} \leq C C_{\mathrm{reg}} h\|\boldsymbol{\delta}\|\|\boldsymbol{\Pi} \boldsymbol{e}\|,
$$

and the first estimate of Theorem (2.2) follows.

Next we consider the case $k=0$. In this case (4.1) is still valid, but we do not have (4.2) since the $L^{2}$-projection into polynomials of degree $k-1$ is no longer defined. Nevertheless, we can still estimate $T_{i}$ for $i=1,2,3,4$ in their form given by (4.1). We provide the details for only $T_{1}$. Applying Cauchy-Schwarz inequality to each term in $T_{1}$ we get

$$
\left|T_{1}\right| \leq\left\|\delta_{T}\right\|\left\|\psi_{\theta}\right\|+\left(\left\|\delta_{T}\right\|+\left\|\Pi_{T} e_{T}\right\|\right)\left\|\delta_{\psi_{\theta}}\right\| .
$$

By Theorem 2.1 we have that

$$
\left|T_{1}\right| \leq\left\|\delta_{T}\right\|\left\|\psi_{\theta}\right\|+\left(\left\|\delta_{T}\right\|+\left\|\Pi_{T} e_{T}\right\|\right) C h\left|\left(\psi_{T}, \psi_{M}, \psi_{\theta}, \psi_{w}\right)\right|_{1},
$$

and, by the elliptic regularity inequality (2.7) we have

$$
\left|T_{1}\right| \leq C C_{\text {reg }}\|\boldsymbol{\delta}\|\|\boldsymbol{\Pi} \boldsymbol{e}\|+C C_{\text {reg }} h\|\boldsymbol{\Pi} \boldsymbol{e}\|^{2} .
$$


Since the remaining terms can be estimated in a similar fashion, we obtain

$$
\|\boldsymbol{\Pi} \boldsymbol{e}\|^{2} \leq C C_{\mathrm{reg}}\|\boldsymbol{\delta}\|\|\boldsymbol{\Pi} \boldsymbol{e}\|+C C_{\mathrm{reg}} h\|\boldsymbol{\Pi} \boldsymbol{e}\|^{2} .
$$

The second estimate of Theorem (2.2) now follows if we assume that $C C_{\mathrm{reg}} h<1$. This completes the proof.

4.2. Step 2: Proof of the duality identity of Lemma 4.1, To prove Lemma 4.1. we begin by obtaining a couple of auxiliary identities. The first is the following.

Lemma 4.2. Let $\left(v_{1}, v_{2}, v_{3}, v_{4}\right) \in\left[H^{1}\left(\Omega_{h}\right)\right]^{4}$ and we take $\mathrm{S}^{t}$ as the stabilization function of the projection $\left(\Pi_{T} v_{1}, \Pi_{M} v_{2}, \Pi_{\theta} v_{3}, \Pi_{\mathcal{W}} v_{4}\right)$. Then

$$
\begin{aligned}
& -\left\langle\widehat{e}_{\theta}-e_{\theta}, \delta_{v_{2}} n\right\rangle_{\partial \Omega_{h}}+\left\langle\widehat{e}_{M}-e_{M}, \delta_{v_{3}} n\right\rangle_{\partial \Omega_{h}} \\
& -\left\langle\widehat{e}_{T}-e_{T}, \delta_{v_{4}} n\right\rangle_{\partial \Omega_{h}}+\left\langle\widehat{e}_{W}-e_{W}, \delta_{v_{1}} n\right\rangle_{\partial \Omega_{h}}=0 .
\end{aligned}
$$

Proof. Let $\Theta$ be the left-hand side of the identity we want to prove, that is,

$$
\Theta:=-\left\langle\left[\begin{array}{c}
\widehat{e}_{\theta}-e_{\theta} \\
\widehat{e}_{T}-e_{T}
\end{array}\right],\left[\begin{array}{c}
\delta_{v_{2}} \\
\delta_{v_{4}}
\end{array}\right] n\right\rangle_{\partial \Omega_{h}}+\left\langle\left[\begin{array}{c}
\widehat{e}_{M}-e_{M} \\
\widehat{e}_{\mathcal{W}}-e_{\mathcal{W}}
\end{array}\right],\left[\begin{array}{c}
\delta_{v_{3}} \\
\delta_{v_{1}}
\end{array}\right] n\right\rangle_{\partial \Omega_{h}} .
$$

Noting that $\widehat{e}_{u}-e_{u}=u_{h}-\widehat{u}_{h}$ for $u=T, M, \theta, w$, and that, by the definition of the numerical traces (2.2b), we have

$$
\left[\begin{array}{c}
\widehat{e}_{\theta}-e_{\theta} \\
\widehat{e}_{T}-e_{T}
\end{array}\right]=\left[\begin{array}{c}
\theta_{h}-\widehat{\theta}_{h} \\
T_{h}-\widehat{T}_{h}
\end{array}\right]=\mathrm{S}\left[\begin{array}{c}
M_{h}-\widehat{M}_{h} \\
w_{h}-\widehat{w}_{h}
\end{array}\right] n
$$

we get

$$
\Theta=-\left\langle\mathrm{S}\left[\begin{array}{c}
M_{h}-\widehat{M}_{h} \\
w_{h}-\widehat{w}_{h}
\end{array}\right],\left[\begin{array}{c}
\delta_{v_{2}} \\
\delta_{v_{4}}
\end{array}\right]\right\rangle_{\partial \Omega_{h}}+\left\langle\left[\begin{array}{c}
M_{h}-\widehat{M}_{h} \\
w_{h}-\widehat{w}_{h}
\end{array}\right], S^{t}\left[\begin{array}{c}
\delta_{v_{2}} \\
\delta_{v_{4}}
\end{array}\right]\right\rangle_{\partial \Omega_{h}}=0
$$

because $\left[\begin{array}{l}\delta_{v_{3}} \\ \delta_{v_{1}}\end{array}\right]=\mathrm{S}^{t}\left[\begin{array}{l}\delta_{v_{2}} \\ \delta_{v_{4}}\end{array}\right] n$, by (2.3e). This completes the proof.

Lemma 4.3. Let $\left(u_{1}, u_{2}, u_{3}, u_{4}\right),\left(v_{1}, v_{2}, v_{3}, v_{4}\right) \in\left[H^{1}\left(\Omega_{h}\right)\right]^{4}$ with the stabilization functions $\mathrm{S}, \mathrm{S}^{t}$, respectively. Then

$$
-\left\langle\delta_{u_{3}}, \delta_{v_{2}} n\right\rangle_{\partial \Omega_{h}}+\left\langle\delta_{u_{2}}, \delta_{v_{3}} n\right\rangle_{\partial \Omega_{h}}-\left\langle\delta_{u_{1}}, \delta_{v_{4}} n\right\rangle_{\partial \Omega_{h}}+\left\langle\delta_{u_{4}}, \delta_{v_{1}} n\right\rangle_{\partial \Omega_{h}}=0 .
$$

Proof. Let $\Theta$ be the left-hand side of the identity we want to prove, that is,

$$
\Theta:=-\left\langle\left[\begin{array}{c}
\delta_{u_{3}} \\
\delta_{u_{1}}
\end{array}\right],\left[\begin{array}{c}
\delta_{v_{2}} \\
\delta_{v_{4}}
\end{array}\right] n\right\rangle_{\partial \Omega_{h}}+\left\langle\left[\begin{array}{c}
\delta_{u_{2}} \\
\delta_{u_{4}}
\end{array}\right],\left[\begin{array}{c}
\delta_{v_{3}} \\
\delta_{v_{1}}
\end{array}\right] n\right\rangle_{\partial \Omega_{h}} .
$$

Since, by (2.3e), we have that $\left[\begin{array}{l}\delta_{u_{3}} \\ \delta_{u_{1}}\end{array}\right]=\mathrm{S}\left[\begin{array}{l}\delta_{u_{2}} \\ \delta_{u_{4}}\end{array}\right] n$, and $\left[\begin{array}{l}\delta_{v_{3}} \\ \delta_{v_{1}}\end{array}\right]=\mathrm{S}^{t}\left[\begin{array}{l}\delta_{v_{2}} \\ \delta_{v_{4}}\end{array}\right] n$, we readily obtain that

$$
\Theta=-\left\langle\mathrm{S}\left[\begin{array}{l}
\delta_{u_{2}} \\
\delta_{u_{4}}
\end{array}\right] n,\left[\begin{array}{l}
\delta_{v_{2}} \\
\delta_{v_{4}}
\end{array}\right] n\right\rangle_{\partial \Omega_{h}}+\left\langle\left[\begin{array}{c}
\delta_{u_{2}} \\
\delta_{u_{4}}
\end{array}\right], \mathrm{S}^{t}\left[\begin{array}{l}
\delta_{v_{2}} \\
\delta_{v_{4}}
\end{array}\right]\right\rangle_{\partial \Omega_{h}}=0 .
$$

This completes the proof.

We are now ready to prove Lemma 4.1 . 
Proof of Lemma 4.1. By the definition of $\mathcal{E}$ and the equations defining the dual solution (2.6), we have

$$
\begin{aligned}
\mathcal{E}= & \left(\Pi_{T} e_{T}, \psi_{\mathfrak{W}}^{\prime}\right)_{\Omega_{h}}-\left(\Pi_{T} e_{T}, \psi_{\theta}\right)_{\Omega_{h}}+\left(\Pi_{T} e_{T}, d^{2} \psi_{T} / G A\right)_{\Omega_{h}} \\
& -\left(\Pi_{M} e_{M}, \psi_{\theta}^{\prime}\right)_{\Omega_{h}}+\left(\Pi_{M} e_{M}, \psi_{M} / E I\right)_{\Omega_{h}} \\
& +\left(\Pi_{\theta} e_{\theta}, \psi_{M}^{\prime}\right)_{\Omega_{h}}-\left(\Pi_{\theta} e_{\theta}, \psi_{T}\right)_{\Omega_{h}}-\left(\Pi_{\mathcal{W}} e_{\mathcal{W}}, \psi_{T}^{\prime}\right)_{\Omega_{h}} .
\end{aligned}
$$

Since, for any pair $\left(e_{u}, \psi_{v}\right)$, we have that

$$
\begin{aligned}
\left(\Pi_{u} e_{u}, \psi_{v}^{\prime}\right)_{\Omega_{h}} & =\left(\Pi_{u} e_{u},\left(\Pi_{v} \psi_{v}\right)^{\prime}\right)_{\Omega_{h}}+\left(\Pi_{u} u, \delta_{\psi_{v}}^{\prime}\right) \\
& =\left(\Pi_{u} e_{u},\left(\Pi_{v} \psi_{v}\right)^{\prime}\right)_{\Omega_{h}}-\left(\left(\Pi_{u} e_{u}\right)^{\prime}, \delta_{\psi_{v}}\right)_{\Omega_{h}}+\left\langle\Pi_{u} u, \delta_{\psi_{v}} n\right\rangle_{\partial \Omega_{h}} \\
& =\left(\Pi_{u} e_{u},\left(\Pi_{v} \psi_{v}\right)^{\prime}\right)_{\Omega_{h}}+\left\langle\Pi_{u} u, \delta_{\psi_{v}} n\right\rangle_{\partial \Omega_{h}},
\end{aligned}
$$

by the orthogonality properties (2.3a) to (2.3d) of the projection. Hence

$$
\begin{aligned}
\mathcal{E}= & \left(\Pi_{T} e_{T},\left(\Pi_{\mathcal{W}} \psi_{\mathcal{W}}\right)^{\prime}\right)_{\Omega_{h}}-\left(\Pi_{T} e_{T}, \psi_{\theta}\right)_{\Omega_{h}}+\left(\Pi_{T} e_{T}, d^{2} \psi_{T} / G A\right)_{\Omega_{h}} \\
& -\left(\Pi_{M} e_{M},\left(\Pi_{\theta} \psi_{\theta}\right)^{\prime}\right)_{\Omega_{h}}+\left(\Pi_{M} e_{M}, \psi_{M} / E I\right)_{\Omega_{h}} \\
& +\left(\Pi_{\theta} e_{\theta},\left(\Pi_{M} \psi_{M}\right)^{\prime}\right)_{\Omega_{h}}-\left(\Pi_{\theta} e_{\theta}, \psi_{T}\right)_{\Omega_{h}}-\left(\Pi_{\mathcal{W}} e_{\mathcal{W}},\left(\Pi_{T} \psi_{T}\right)^{\prime}\right)_{\Omega_{h}} \\
& +\left\langle\Pi_{T} e_{T}, \delta_{\psi_{W}} n\right\rangle_{\partial \Omega_{h}}-\left\langle\Pi_{M} e_{M}, \delta_{\psi_{\theta}}\right\rangle_{\partial \Omega_{h}} \\
& +\left\langle\Pi_{\theta} e_{\theta}, \delta_{\psi_{M}} n\right\rangle_{\partial \Omega_{h}}-\left\langle\Pi_{\mathcal{W}} e_{\mathcal{W}}, \delta_{\psi_{T}} n\right\rangle_{\partial \Omega_{h}} .
\end{aligned}
$$

Taking $\left(v_{1}, v_{2}, v_{3}, v_{4}\right)=\left(-\Pi_{T} \psi_{T}, \Pi_{M} \psi_{M},-\Pi_{\theta} \psi_{\theta}, \Pi_{\mathfrak{W}} \psi_{\mathcal{W}}\right)$ in the error equations (2.4) and carrying out some very simple algebraic manipulations, we obtain

$$
\begin{aligned}
& \mathcal{E}=H+\left(\delta_{T}, \Pi_{\theta} \psi_{\theta}\right)_{\Omega_{h}} \quad-\left(\Pi_{T} e_{T}, \delta_{\psi_{\theta}}\right)_{\Omega_{h}} \\
& +\left(\Pi_{T} e_{T}, d^{2} \delta_{\psi_{T}} / G A\right)_{\Omega_{h}}-\left(\delta_{T}, d^{2} \Pi_{T} \psi_{T} / G A\right)_{\Omega_{h}} \\
& +\left(\Pi_{M} e_{M}, \delta_{\psi_{M}} / E I\right)_{\Omega_{h}}-\left(\delta_{M}, \Pi_{M} \psi_{M} / E I\right)_{\Omega_{h}} \\
& +\left(\delta_{\theta}, \Pi_{T} \psi_{T}\right)_{\Omega_{h}} \quad-\left(\Pi_{\theta} e_{\theta}, \delta_{\psi_{T}}\right)_{\Omega_{h}}
\end{aligned}
$$

where

$$
\begin{aligned}
& H:=\left\langle\widehat{e}_{T}, \Pi_{\mathcal{W}} \psi_{\mathcal{W}} n\right\rangle_{\partial \Omega_{h}}+\left\langle\Pi_{T} e_{T}, \delta_{\psi_{W}} n\right\rangle_{\partial \Omega_{h}} \\
& -\left\langle\widehat{e}_{M}, \Pi_{\theta} \psi_{\theta} n\right\rangle_{\partial \Omega_{h}} \quad-\left\langle\Pi_{M} e_{M}, \delta_{\psi_{\theta}} n\right\rangle_{\partial \Omega_{h}} \\
& +\left\langle\widehat{e}_{\theta}, \Pi_{M} \psi_{M} n\right\rangle_{\partial \Omega_{h}} \quad+\left\langle\Pi_{T} e_{\theta}, \delta_{\psi_{M}} n\right\rangle_{\partial \Omega_{h}} \\
& -\left\langle\widehat{e}_{\mathcal{W}}, \Pi_{T} \psi_{T} n\right\rangle_{\partial \Omega_{h}} \quad-\left\langle\Pi_{\mathcal{W}} e_{\mathcal{W}}, \delta_{\psi_{T}} n\right\rangle_{\partial \Omega_{h}} .
\end{aligned}
$$

It remains to show that $H=0$.

Since $\psi_{M}$ and $\psi_{\mathcal{W}}$ are single-valued on $\mathscr{E}_{h}$, and $\psi_{\mathfrak{W}}=0$ on $\partial \Omega$, we can take $\mathrm{m}:=\psi_{M}$ and $\mathrm{w}:=\psi_{\mathrm{w}}$ in the error equations (2.4e) and (2.4f), respectively, to get

$$
\left\langle\widehat{e}_{\theta}, \psi_{M} n\right\rangle_{\partial \Omega_{h}}=\left\langle\widehat{e}_{T}, \psi_{\mathcal{W}} n\right\rangle_{\partial \Omega_{h}}=0 .
$$

Moreover, since $\widehat{e}_{M}$ and $\widehat{e}_{\mathcal{W}}$ are single-valued on $\mathscr{E}_{h}$, and $\widehat{e}_{\mathcal{W}}=0, \psi_{\theta}=0$ on $\partial \Omega_{h}$, we have

$$
\left\langle\widehat{e}_{M}, \psi_{\theta} n\right\rangle_{\partial \Omega_{h}}=\left\langle\widehat{e}_{\mathcal{W}}, \psi_{T} n\right\rangle_{\partial \Omega_{h}}=0 .
$$


This implies that

$$
\begin{aligned}
& H=\left\langle\widehat{e}_{T},\left(\Pi_{\mathcal{W}} \psi_{\mathcal{W}}-\psi_{\mathfrak{W}}\right) n\right\rangle_{\partial \Omega_{h}}+\left\langle\Pi_{T} e_{T}, \delta_{\psi_{\mathcal{W}}} n\right\rangle_{\partial \Omega_{h}} \\
& -\left\langle\widehat{e}_{M},\left(\Pi_{\theta} \psi_{\theta}-\psi_{\theta}\right) n\right\rangle_{\partial \Omega_{h}}-\left\langle\Pi_{M} e_{M}, \delta_{\psi_{\theta}} n\right\rangle_{\partial \Omega_{h}} \\
& +\left\langle\widehat{e}_{\theta},\left(\Pi_{M} \psi_{M}-\psi_{M}\right) n\right\rangle_{\partial \Omega_{h}}+\left\langle\Pi_{\theta} e_{\theta}, \delta_{\psi_{M}} n\right\rangle_{\partial \Omega_{h}} \\
& -\left\langle\widehat{e}_{\mathcal{W}},\left(\Pi_{T} \psi_{T}-\psi_{T}\right) n\right\rangle_{\partial \Omega_{h}}-\left\langle\Pi_{\mathcal{W}} e_{\mathcal{W}}, \delta_{\psi_{T}} n\right\rangle_{\partial \Omega_{h}} \\
& =-\left\langle\widehat{e}_{T}-\Pi_{T} e_{T}, \delta_{\psi_{W}} n\right\rangle_{\partial \Omega_{h}} \quad+\left\langle\widehat{e}_{M}-\Pi_{M} e_{M}, \delta_{\psi_{\theta}} n\right\rangle_{\partial \Omega_{h}} \\
& -\left\langle\widehat{e}_{\theta}-\Pi_{\theta} e_{\theta}, \delta_{\psi_{M}} n\right\rangle_{\partial \Omega_{h}}+\left\langle\widehat{e}_{\mathcal{W}}-\Pi_{\mathcal{W}} e_{\mathcal{W}}, \delta_{\psi_{T}} n\right\rangle_{\partial \Omega_{h}} \\
& =H_{1}+H_{2} \text {, }
\end{aligned}
$$

where

$$
\begin{aligned}
& H_{1}=-\left\langle\widehat{e}_{T}-e_{T}, \delta_{\psi_{W}} n\right\rangle_{\partial \Omega_{h}}+\left\langle\widehat{e}_{M}-e_{M}, \delta_{\psi_{\theta}} n\right\rangle_{\partial \Omega_{h}} \\
& -\left\langle\widehat{e}_{\theta}-e_{\theta}, \delta_{\psi_{M}} n\right\rangle_{\partial \Omega_{h}}+\left\langle\widehat{e}_{\mathcal{W}}-e_{\mathcal{W}}, \delta_{\psi_{T}} n\right\rangle_{\partial \Omega_{h}}, \\
& H_{2}=-\left\langle\delta_{T}, \delta_{\psi_{W}} n\right\rangle_{\partial \Omega_{h}} \quad+\left\langle\delta_{M}, \delta_{\psi_{\theta}} n\right\rangle_{\partial \Omega_{h}} \\
& -\left\langle\delta_{\theta}, \delta_{\psi_{M}} n\right\rangle_{\partial \Omega_{h}} \quad+\left\langle\delta_{\mathcal{W}}, \delta_{\psi_{T}} n\right\rangle_{\partial \Omega_{h}} .
\end{aligned}
$$

But $H_{1}=0$ by Lemma 4.2 with $\left(v_{1}, v_{2}, v_{3}, v_{4}\right)=\left(\psi_{T}, \psi_{M}, \psi_{\theta}, \psi_{\mathcal{W}}\right)$, and $H_{2}=0$ by Lemma 4.3 with $\left(u_{1}, u_{2}, u_{3}, u_{4}\right)=(T, M, \theta, w)$ and $\left(v_{1}, v_{2}, v_{3}, v_{4}\right)=\left(\psi_{T}, \psi_{M}, \psi_{\theta}, \psi_{\mathfrak{w}}\right)$. This completes the proof.

\section{Error of the numerical traces: Proof of Theorem 2.4}

To prove this theorem we proceed in two steps. In the first, we obtain representation formulas for the errors in the numerical traces. In the second, we use approximation results to estimate them. We prove the result only for $k \geq 1$; the proof for the case $k=0$ is not difficult.

5.1. Step 1: Representation of the errors. The following lemma provides a representation formula for the errors in the numerical traces.

Lemma 5.1. Let $x_{i} \in \mathscr{E}_{h}$ be an arbitrary node and let $\Phi_{T, x_{i}}^{u}, \Phi_{M, x_{i}}^{u}, \Phi_{\theta, x_{i}}^{u}, \Phi_{\mathfrak{W}, x_{i}}^{u}$, for $u=T, M, \theta$, or $w$, be the functions defined by (2.8a), (2.8b), and (2.8c). Then

$$
\widehat{e}_{u}\left(x_{i}\right)=\Gamma_{1}^{u}\left(x_{i}\right)+\Gamma_{2}^{u}\left(x_{i}\right)
$$

where

$$
\begin{aligned}
\Gamma_{1}^{u}\left(x_{i}\right)= & \left(\mathfrak{W}^{\prime}-\left(\mathfrak{w}^{\prime}\right)_{k-1}, \Phi_{T, x_{i}}^{u}-\Pi_{T} \Phi_{T, x_{i}}^{u}\right)_{\Omega_{h}} \\
& -\left(\theta^{\prime}-\left(\theta^{\prime}\right)_{k-1}, \Phi_{M, x_{i}}^{u}-\Phi_{M, x_{i}} \Phi_{M, x_{i}}^{u}\right)_{\Omega_{h}} \\
& +\left(M^{\prime}-\left(M^{\prime}\right)_{k-1}, \Phi_{\theta, x_{i}}^{u}-\Pi_{\theta} \Phi_{\theta, x_{i}}^{u}\right)_{\Omega_{h}} \\
& -\left(T^{\prime}-\left(T^{\prime}\right)_{k-1}, \Phi_{\mathcal{W}, x_{i}}^{u}-\Pi_{\mathfrak{W}} \Phi_{\mathcal{W}, x_{i}}^{u}\right)_{\Omega_{h}}, \\
\Gamma_{2}^{u}\left(x_{i}\right)= & \left(e_{M} / E I, \Phi_{M, x_{i}}^{u}-\Pi_{M} \Phi_{M, x_{i}}^{u}\right)_{\Omega_{h}}-\left(e_{\theta}, \Phi_{T, x_{i}}^{u}-\Pi_{T} \Phi_{T, x_{i}}^{u}\right)_{\Omega_{h}} \\
& +\left(d^{2} e_{T} / G A, \Phi_{T, x_{i}}^{u}-\Pi_{T} \Phi_{T, x_{i}}^{u}\right)_{\Omega_{h}}-\left(e_{T}, \Phi_{\theta, x_{i}}^{u}-\Pi_{\theta} \Phi_{\theta, x_{i}}^{u}\right)_{\Omega_{h}} .
\end{aligned}
$$

To prove this lemma we need an auxiliary result which establishes a relation between the errors in the numerical traces and the Green's functions.

Lemma 5.2. Set

$$
\Theta_{i}^{u}:=\left\langle\widehat{e}_{\mathcal{W}}, \Phi_{T, x_{i}}^{u} n\right\rangle_{\partial \Omega_{h}}-\left\langle\widehat{e}_{\theta}, \Phi_{M, x_{i}}^{u} n\right\rangle_{\partial \Omega_{h}}+\left\langle\widehat{e}_{M}, \Phi_{\theta, x_{i}}^{u} n\right\rangle_{\partial \Omega_{h}}-\left\langle\widehat{e}_{T}, \Phi_{\mathcal{W}, x_{i}}^{u} n\right\rangle_{\partial \Omega_{h}} .
$$


Then, we have $\Theta_{i}^{u}=\Theta_{i, 1}^{u}+\Theta_{i, 2}^{u}+\Theta_{i, 3}^{u}$ where

$$
\begin{array}{rlr}
\Theta_{i, 1}^{u}= & \left\langle\widehat{e}_{\mathcal{W}}-e_{\mathcal{W}},\left(\Phi_{T, x_{i}}^{u}-v_{1}\right) n\right\rangle_{\partial \Omega_{h}} & -\left\langle\widehat{e}_{\theta}-e_{\theta},\left(\Phi_{M, x_{i}}^{u}-v_{2}\right) n\right\rangle_{\partial \Omega_{h}} \\
& +\left\langle\widehat{e}_{M}-e_{M},\left(\Phi_{\theta, x_{i}}^{u}-v_{3}\right) n\right\rangle_{\partial \Omega_{h}}-\left\langle\widehat{e}_{T}-e_{T},\left(\Phi_{\mathcal{W}, x_{i}}^{u}-v_{4}\right) n\right\rangle_{\partial \Omega_{h}}, \\
\Theta_{i, 2}^{u}= & \left(e_{\mathfrak{W}}^{\prime}, \Phi_{T, x_{i}}^{u}-v_{1}\right)_{\Omega_{h}} & -\left(e_{\theta}^{\prime}, \Phi_{M, x_{i}}^{u}-v_{2}\right)_{\Omega_{h}} \\
& +\left(e_{M}^{\prime}, \Phi_{\theta, x_{i}}^{u}-v_{3}\right)_{\Omega_{h}} & -\left(e_{T}^{\prime}, \Phi_{\mathcal{W}, x_{i}}^{u}-v_{4}\right)_{\Omega_{h}}, \\
\Theta_{i, 3}^{u}=\left(e_{M} / E I, \Phi_{M, x_{i}}^{u}-v_{2}\right)_{\Omega_{h}} & -\left(e_{\theta}, \Phi_{T, x_{i}}^{u}-v_{1}\right)_{\Omega_{h}} \\
& +\left(d^{2} e_{T} / G A, \Phi_{T, x_{i}}^{u}-v_{1}\right)_{\Omega_{h}} & -\left(e_{T}, \Phi_{\theta, x_{i}}^{u}-v_{3}\right)_{\Omega_{h}},
\end{array}
$$

for $\left(v_{1}, v_{2}, v_{3}, v_{4}\right) \in\left[V_{h}^{k}\right]^{4}$.

Proof. Adding and subtracting the term

$$
\left\langle\widehat{e}_{W}, v_{1} n\right\rangle_{\partial \Omega_{h}}-\left\langle\widehat{e}_{\theta}, v_{2} n\right\rangle_{\partial \Omega_{h}}+\left\langle\widehat{e}_{M}, v_{3} n\right\rangle_{\partial \Omega_{h}}-\left\langle\widehat{e}_{T}, v_{4} n\right\rangle_{\partial \Omega_{h}}
$$

to the original expression for $\Theta_{i}^{u}$, we see that

$$
\begin{aligned}
\Theta_{i}^{u}= & \left\langle\widehat{e}_{\mathcal{W}},\left(\Phi_{T, x_{i}}^{u}-v_{1}\right) n\right\rangle_{\partial \Omega_{h}}-\left\langle\widehat{e}_{\theta},\left(\Phi_{M, x_{i}}^{u}-v_{2}\right) n\right\rangle_{\partial \Omega_{h}} \\
& +\left\langle\widehat{e}_{M},\left(\Phi_{\theta, x_{i}}^{u}-v_{3}\right) n\right\rangle_{\partial \Omega_{h}}-\left\langle\widehat{e}_{T},\left(\Phi_{W, x_{i}}^{u}-v_{4}\right) n\right\rangle_{\partial \Omega_{h}} \\
& +\left\langle\widehat{e}_{W}, v_{1} n\right\rangle_{\partial \Omega_{h}}-\left\langle\widehat{e}_{\theta}, v_{2} n\right\rangle_{\partial \Omega_{h}} \\
& +\left\langle\widehat{e}_{M}, v_{3} n\right\rangle_{\partial \Omega_{h}}-\left\langle\widehat{e}_{T}, v_{4} n\right\rangle_{\partial \Omega_{h}} .
\end{aligned}
$$

Rewriting the last four terms above by using the error equations (2.4ad - 2.4d), we obtain

$$
\begin{aligned}
\Theta_{i}^{u}= & \left\langle\widehat{e}_{\mathcal{W}},\left(\Phi_{T, x_{i}}^{u}-v_{1}\right) n\right\rangle_{\partial \Omega_{h}}-\left\langle\widehat{e}_{\theta},\left(\Phi_{M, x_{i}}^{u}-v_{2}\right) n\right\rangle_{\partial \Omega_{h}} \\
& +\left\langle\widehat{e}_{M},\left(\Phi_{\theta, x_{i}}^{u}-v_{3}\right) n\right\rangle_{\partial \Omega_{h}}-\left\langle\widehat{e}_{T},\left(\Phi_{\mathcal{W}, x_{i}}^{u}-v_{4}\right) n\right\rangle_{\partial \Omega_{h}} \\
& +\left(e_{\mathcal{W}}, v_{1}^{\prime}\right)_{\Omega_{h}}-\left(e_{\theta}, v_{2}^{\prime}\right)_{\Omega_{h}}+\left(e_{M}, v_{3}^{\prime}\right)_{\Omega_{h}}-\left(e_{T}, v_{4}^{\prime}\right)_{\Omega_{h}} \\
& +\left(e_{\theta}, v_{1}\right)_{\Omega_{h}}-\left(d^{2} e_{T} / G A, v_{1}\right)_{\Omega_{h}}-\left(e_{M} / E I, v_{2}\right)_{\Omega_{h}}+\left(e_{T}, v_{3}\right)_{\Omega_{h}} .
\end{aligned}
$$

Note that, by the definition of the Green's functions, we have

$$
\begin{aligned}
& \left(e_{\mathcal{W}},\left(\Phi_{T, x_{i}}^{u}\right)^{\prime}\right)_{\Omega_{h}}=0 \\
& \left(e_{\theta},\left(\Phi_{M, x_{i}}^{u}\right)^{\prime}\right)_{\Omega_{h}}=\left(e_{\theta}, \Phi_{T, x_{i}}^{u}\right)_{\Omega_{h}}, \\
& \left(e_{M},\left(\Phi_{\theta, x_{i}}^{u}\right)^{\prime}\right)_{\Omega_{h}}=\left(e_{M}, \Phi_{M, x_{i}}^{u} / E I\right)_{\Omega_{h}}, \\
& \left(e_{T},\left(\Phi_{\mathcal{W}, x_{i}}^{u}\right)^{\prime}\right)_{\Omega_{h}}=\left(e_{T}, \Phi_{\theta, x_{i}}^{u}-d^{2} \Phi_{T, x_{i}}^{u} / G A\right)_{\Omega_{h}} .
\end{aligned}
$$

Inserting these equations into the last expression for $\Theta_{i}^{u}$, and rearranging terms, we obtain

$$
\begin{aligned}
\Theta_{i}^{u}=\Theta_{i, 3}^{u} & +\left\langle\widehat{e}_{\mathfrak{W}},\left(\Phi_{T, x_{i}}^{u}-v_{1}\right) n\right\rangle_{\partial \Omega_{h}}-\left\langle\widehat{e}_{\theta},\left(\Phi_{M, x_{i}}^{u}-v_{2}\right) n\right\rangle_{\partial \Omega_{h}} \\
& +\left\langle\widehat{e}_{M},\left(\Phi_{\theta, x_{i}}^{u}-v_{3}\right) n\right\rangle_{\partial \Omega_{h}}-\left\langle\widehat{e}_{T},\left(\Phi_{\mathcal{W}, x_{i}}^{u}-v_{4}\right) n\right\rangle_{\partial \Omega_{h}} \\
& -\left(e_{\mathcal{W}},\left(\Phi_{T, x_{i}}^{u}-v_{1}\right)^{\prime}\right)_{\Omega_{h}}+\left(e_{\theta},\left(\Phi_{M, x_{i}}^{u}-v_{2}\right)^{\prime}\right)_{\Omega_{h}} \\
& -\left(e_{M},\left(\Phi_{\theta, x_{i}}^{u}-v_{3}\right)^{\prime}\right)_{\Omega_{h}}+\left(e_{T},\left(\Phi_{\mathcal{W}, x_{i}}^{u}-v_{4}\right)^{\prime}\right)_{\Omega_{h}} .
\end{aligned}
$$

It remains to show that

$$
\begin{aligned}
\Theta_{i, 1}^{u}+\Theta_{i, 2}^{u}= & \left\langle\widehat{e}_{\mathcal{W}},\left(\Phi_{T, x_{i}}^{u}-v_{1}\right) n\right\rangle_{\partial \Omega_{h}}-\left\langle\widehat{e}_{\theta},\left(\Phi_{M, x_{i}}^{u}-v_{2}\right) n\right\rangle_{\partial \Omega_{h}} \\
& +\left\langle\widehat{e}_{M},\left(\Phi_{\theta, x_{i}}^{u}-v_{3}\right) n\right\rangle_{\partial \Omega_{h}}-\left\langle\widehat{e}_{T},\left(\Phi_{\mathcal{W}, x_{i}}^{u}-v_{4}\right) n\right\rangle_{\partial \Omega_{h}} \\
& -\left(e_{\mathcal{W}},\left(\Phi_{T, x_{i}}^{u}-v_{1}\right)^{\prime}\right)_{\Omega_{h}}+\left(e_{\theta},\left(\Phi_{M, x_{i}}^{u}-v_{2}\right)^{\prime}\right)_{\Omega_{h}} \\
& -\left(e_{M},\left(\Phi_{\theta, x_{i}}^{u}-v_{3}\right)^{\prime}\right)_{\Omega_{h}}+\left(e_{T},\left(\Phi_{\mathcal{W}, x_{i}}^{u}-v_{4}\right)^{\prime}\right)_{\Omega_{h}} .
\end{aligned}
$$


This follows by integrating by parts on each of the last four terms. This completes the proof.

We are now ready to prove our representation result.

Proof of Lemma 5.1. We begin by noting that, by the definition of the Green's functions, (2.8b and (2.8c), we have

$$
\Theta_{i}^{u}=\widehat{e}_{u}\left(x_{i}\right)
$$

On the other hand, setting

$$
\left(v_{1}, v_{2}, v_{3}, v_{4}\right)=\left(\Pi_{T} \Phi_{T, x_{i}}^{u}, \Pi_{M} \Phi_{M, x_{i}}^{u}, \Pi_{\theta} \Phi_{\theta, x_{i}}^{u}, \Pi_{\mathcal{W}} \Phi_{\mathcal{W}, x_{i}}^{u}\right)
$$

in Lemma 5.2. we obtain

$$
\widehat{e}_{u}\left(x_{i}\right)=\Theta_{i, 1}^{u}+\Theta_{i, 2}^{u}+\Theta_{i, 3}^{u}
$$

with

$$
\begin{aligned}
& \Theta_{i, 1}^{u}=\left\langle\widehat{e}_{\mathcal{W}}-e_{\mathcal{W}},\left(\Phi_{T, x_{i}}^{u}-\Pi_{T} \Phi_{T, x_{i}}^{u}\right) n\right\rangle_{\partial \Omega_{h}}-\left\langle\widehat{e}_{\theta}-e_{\theta},\left(\Phi_{M, x_{i}}^{u}-\Pi_{M} \Phi_{M, x_{i}}^{u}\right) n\right\rangle_{\partial \Omega_{h}} \\
& +\left\langle\widehat{e}_{M}-e_{M},\left(\Phi_{\theta, x_{i}}^{u}-\Pi_{\theta} \Phi_{\theta, x_{i}}^{u}\right) n\right\rangle_{\partial \Omega_{h}}-\left\langle\widehat{e}_{T}-e_{T},\left(\Phi_{\mathcal{W}, x_{i}}^{u}-\Pi_{\mathcal{W}} \Phi_{\mathcal{W}, x_{i}}^{u}\right) n\right\rangle_{\partial \Omega_{h}}, \\
& \Theta_{i, 2}^{u}=\left(e_{\mathcal{W}}^{\prime}, \Phi_{T, x_{i}}^{u}-\Pi_{T} \Phi_{T, x_{i}}^{u}\right)_{\Omega_{h}} \quad-\left(e_{\theta}^{\prime}, \Phi_{M, x_{i}}^{u}-\Pi_{M} \Phi_{M, x_{i}}^{u}\right)_{\Omega_{h}} \\
& +\left(e_{M}^{\prime}, \Phi_{\theta, x_{i}}^{u}-\Pi_{\theta} \Phi_{\theta, x_{i}}^{u}\right)_{\Omega_{h}} \quad-\left(e_{T}^{\prime}, \Phi_{\mathcal{W}, x_{i}}^{u}-\Pi_{\mathcal{W}} \Phi_{\mathcal{W}, x_{i}}^{u}\right)_{\Omega_{h}}, \\
& \Theta_{i, 3}^{u}=\left(e_{M} / E I, \Phi_{M, x_{i}}^{u}-\Pi_{M} \Phi_{M, x_{i}}^{u}\right)_{\Omega_{h}} \quad-\left(e_{\theta}, \Phi_{T, x_{i}}^{u}-\Pi_{T} \Phi_{T, x_{i}}^{u}\right)_{\Omega_{h}} \\
& +\left(d^{2} e_{T} / G A, \Phi_{T, x_{i}}^{u}-\Pi_{T} \Phi_{T, x_{i}}^{u}\right)_{\Omega_{h}} \quad-\left(e_{T}, \Phi_{\theta, x_{i}}^{u}-\Pi_{\theta} \Phi_{\theta, x_{i}}^{u}\right)_{\Omega_{h}},
\end{aligned}
$$

Clearly,

$$
\Theta_{i, 3}^{u}=\Gamma_{2}^{u}\left(x_{i}\right)
$$

By Lemma 4.2 with $\left(v_{1}, v_{2}, v_{3}, v_{4}\right)=\left(\Phi_{T, x_{i}}, \Phi_{M, x_{i}}, \Phi_{\theta, x_{i}}, \Phi_{\mathcal{W}, x_{i}}\right)$ we have that

$$
\Theta_{i, 1}^{u}=0 \text {. }
$$

By the orthogonality property, (2.3), of the projection we have

$$
\begin{aligned}
\Theta_{i, 2}^{u}= & \left(e_{\mathcal{W}}^{\prime}-\left(e_{\mathcal{W}}^{\prime}\right)_{k-1}, \Phi_{T, x_{i}}^{u}-\Pi_{T} \Phi_{T, x_{i}}^{u}\right)_{\Omega_{h}} \\
& -\left(e_{\theta}^{\prime}-\left(e_{\theta}^{\prime}\right)_{k-1}, \Phi_{M, x_{i}}^{u}-\Pi_{M} \Phi_{M, x_{i}}^{u}\right)_{\Omega_{h}} \\
& +\left(e_{M}^{\prime}-\left(e_{M}^{\prime}\right)_{k-1}, \Phi_{\theta, x_{i}}^{u}-\Pi_{\theta} \Phi_{\theta, x_{i}}^{u}\right)_{\Omega_{h}} \\
& -\left(e_{T}^{\prime}-\left(e_{T}^{\prime}\right)_{k-1}, \Phi_{\mathcal{W}, x_{i}}^{u}-\Pi_{\mathcal{W}} \Phi_{\mathcal{W}, x_{i}}^{u}\right)_{\Omega_{h}} .
\end{aligned}
$$

Since

$$
\begin{aligned}
e_{u}^{\prime}-\left(e_{u}^{\prime}\right)_{k-1} & =\left(u^{\prime}-u_{h}^{\prime}\right)-\left(u^{\prime}-u_{h}^{\prime}\right)_{k-1} \\
& =u^{\prime}-\left(u^{\prime}\right)_{k-1}+\left(u_{h}^{\prime}\right)_{k-1}-u_{h}^{\prime} \\
& =u^{\prime}-\left(u^{\prime}\right)_{k-1},
\end{aligned}
$$

we see that

$$
\Theta_{i, 2}^{u}=\Gamma_{1}^{u}\left(x_{i}\right) .
$$

The result now follows from (5.1), (5.2), (5.3), and (5.4). 
5.2. Step 2: Proof of Theorem 2.4, We are now ready to prove Theorem 2.4 By Lemma 5.1 we have that

$$
\left|\widehat{e}_{u}\left(x_{i}\right)\right| \leq\left|\Gamma_{1}^{u}\left(x_{i}\right)\right|+\left|\Gamma_{2}^{u}\left(x_{i}\right)\right|
$$

where $\Gamma_{1}^{u}\left(x_{i}\right)=T_{1}+T_{2}+T_{3}+T_{4}$ with

$$
\begin{aligned}
& T_{1}=\left(\mathfrak{w}^{\prime}-\left(\mathfrak{w}^{\prime}\right)_{k-1}, \Phi_{T, x_{i}}^{u}-\Pi_{T} \Phi_{T, x_{i}}^{u}\right)_{\Omega_{h}}, \\
& T_{2}=-\left(\theta^{\prime}-\left(\theta^{\prime}\right)_{k-1}, \Phi_{M, x_{i}}^{u}-\Pi_{M} \Phi_{M, x_{i}}^{u}\right)_{\Omega_{h}}, \\
& T_{3}=\left(M^{\prime}-\left(M^{\prime}\right)_{k-1}, \Phi_{\theta, x_{i}}^{u}-\Pi_{\theta} \Phi_{\theta, x_{i}}^{u}\right)_{\Omega_{h}}, \\
& T_{4}=-\left(T^{\prime}-\left(T^{\prime}\right)_{k-1}, \Phi_{\mathcal{W}, x_{i}}^{u}-\Pi_{\mathcal{W}} \Phi_{\mathcal{W}, x_{i}}^{u}\right)_{\Omega_{h}},
\end{aligned}
$$

and $\Gamma_{2}^{u}\left(x_{i}\right)=S_{1}+S_{2}+S_{3}+S_{4}$ with

$$
\begin{aligned}
& S_{1}=\left(e_{M} / E I, \Phi_{M, x_{i}}^{u}-\Pi_{M} \Phi_{M, x_{i}}^{u}\right)_{\Omega_{h}}, \\
& S_{2}=-\left(e_{\theta}, \Phi_{T, x_{i}}^{u}-\Pi_{T} \Phi_{T, x_{i}}^{u}\right)_{\Omega_{h}}, \\
& S_{3}=\left(d^{2} e_{T} / G A, \Phi_{T, x_{i}}^{u}-\Pi_{T} \Phi_{T, x_{i}}^{u}\right)_{\Omega_{h}}, \\
& S_{4}=-\left(e_{T}, \Phi_{\theta, x_{i}}^{u}-\Pi_{\theta} \Phi_{\theta, x_{i}}^{u}\right)_{\Omega_{h}} .
\end{aligned}
$$

Let us estimate the term $T_{1}$. By the approximation properties of the $L^{2}$-projection and Theorem 2.1, we get

$$
\begin{aligned}
T_{1} & \leq\left\|w^{\prime}-\left(w^{\prime}\right)_{k-1}\right\| \cdot\left\|\Phi_{T, x_{i}}^{u}-\Pi_{T} \Phi_{T, x_{i}}^{u}\right\| \\
& \leq C h^{s}\left|\mathfrak{w}^{\prime}\right|_{s}\left\|\boldsymbol{\delta}_{\boldsymbol{i}}^{u}\right\| \\
& \leq C h^{s}|(T, M, \theta, w)|_{s+1}\left\|\boldsymbol{\delta}_{i}^{u}\right\|,
\end{aligned}
$$

for any $s \in[1, k]$. We estimate the terms $T_{2}, T_{2}$, and $T_{3}$ in a similar fashion and obtain

$$
\left|\Gamma_{1}^{u}\left(x_{i}\right)\right| \leq C h^{s}|(T, M, \theta, w)|_{s+1} \mid\left\|\boldsymbol{\delta}_{i}^{u}\right\| .
$$

Next, we obtain an estimate on $\Gamma_{2}^{u}\left(x_{i}\right)$. We only show how to estimate $S_{1}$ since the remaining terms in $\Gamma_{2}^{u}\left(x_{i}\right)$ can be estimated similarly. By Theorem 2.3, and Lemma 2.1, we get

$$
S_{1} \leq\left\|e_{M} / E I\right\| \cdot\left\|\Phi_{M, x_{i}}^{u}-\Pi_{M} \Phi_{T, x_{i}}^{u}\right\| \leq C\|\boldsymbol{e}\|\left\|\boldsymbol{\delta}_{i}^{u}\right\| .
$$

This implies that

$$
\left|\Gamma_{2}^{u}\left(x_{i}\right)\right| \leq C\|\boldsymbol{e}\|\left\|\boldsymbol{\delta}_{i}^{u}\right\| .
$$

Inserting the estimates of $\left|\Gamma_{1}^{u}\left(x_{i}\right)\right|$ and $\left|\Gamma_{2}^{u}\left(x_{i}\right)\right|$ with $s=k$ into (5.5) completes the proof of Theorem 2.4.

\section{Numerical Results}

In this section, we display numerical results to verify our theoretical findings. We solve the equations (1.1a) with $q(x)=e^{x}, \quad(E I)(x)=e^{x}, \quad(G A)(x)=e^{-x}$, together with the boundary conditions (1.1b) $w(0)=w(1)=\theta(0)=\theta(1)=0$. We take $\mathrm{S}:=\left[\begin{array}{cc}\alpha_{\theta} & \tau \\ \tau & -\alpha_{T}\end{array}\right]$ to be constant on $\partial \Omega_{h}$.

We display our numerical results in Tables 6.1 and 6.2 In Table 6.1 we present a history of convergence study for the projection of the errors, namely,

$$
\Pi e:=\left(\Pi_{T} e_{T}, \Pi_{M} e_{M}, \Pi_{\theta} e_{\theta}, \Pi_{\mathcal{W}} e_{\mathcal{W}}\right) .
$$

Therein, "mesh $=i$ " means we employed a uniform mesh with $2^{i}$ elements to obtain the results of that particular row of the table. For polynomial degrees $k=0,1,2,3$ 
we display the error $\|\boldsymbol{\Pi} \boldsymbol{e}\|$. We also display numerical orders of convergence which are computed as follows. Let $e_{u}(i)$ denote the error where a mesh with $2^{i}$ elements has been employed to obtain the HDG solution. As usual, the order of convergence, $r_{i}$, at level $i$ is defined as $r_{i}:=\log \left(e_{u}(i-1) / e_{u}(i)\right) / \log 2$. Observe that the results displayed in Table 6.1 validate the superconvergence of order $k+2$ for $k \geq 1$, and optimal convergence for $k=0$, predicted by Theorem 2.2 .

In Table 6.2 we carry out a similar study for the errors in the numerical traces. We display the error

$$
\|\widehat{e}\|_{\infty}:=\max _{u \in\{T, M, \theta, \mathfrak{w}\}}\left(\max _{x \in \mathscr{E}_{h}}\left|\left(u-\widehat{u}_{h}\right)(x)\right|\right),
$$

and its order of convergence. We see that the $2 k+1$ order superconvergence of the numerical traces predicted by Theorem 2.4 is verified.

In these examples we took the thickness parameter $d=10^{-2}$, but let us note that in the numerical experiments which we do not report here that we observed similar results and exactly the same convergence orders when we took $d=10^{-8}$.

We do not report here any numerical results for validating the optimal order $L^{2}$ convergence of the error because these results have already been presented in 4 .

TABLE 6.1. History of convergence of the projection of the error.

\begin{tabular}{|c|c|c|c|c|c|c|c|c|}
\hline \multirow[b]{2}{*}{ mesh } & \multicolumn{2}{|c|}{$k=0$} & \multicolumn{2}{|c|}{$k=1$} & \multicolumn{2}{|c|}{$k=2$} & \multicolumn{2}{|c|}{$k=3$} \\
\hline & $\|\Pi e\|$ & order & $\|\Pi e\|$ & order & $\|\Pi e\|$ & order & $\|\Pi e\|$ & order \\
\hline & \multicolumn{8}{|c|}{$\alpha_{\theta} \equiv 0, \alpha_{T} \equiv 0, \tau \equiv 1$} \\
\hline 3 & $1.44 \mathrm{E}-01$ & 0.94 & $4.56 \mathrm{E}-04$ & 2.95 & $3.87 \mathrm{E}-07$ & 4.54 & $1.78 \mathrm{E}-09$ & 4.97 \\
\hline 4 & $7.36 \mathrm{E}-02$ & 0.97 & $5.81 \mathrm{E}-05$ & 2.97 & $2.06 \mathrm{E}-08$ & 4.24 & $5.62 \mathrm{E}-11$ & 4.99 \\
\hline 5 & $3.72 \mathrm{E}-02$ & 0.98 & $7.34 \mathrm{E}-06$ & 2.99 & $1.22 \mathrm{E}-09$ & 4.07 & $1.76 \mathrm{E}-12$ & 4.99 \\
\hline \multirow[t]{2}{*}{6} & $1.87 \mathrm{E}-02$ & 0.99 & $9.22 \mathrm{E}-07$ & 2.99 & $7.54 \mathrm{E}-11$ & 4.02 & $5.50 \mathrm{E}-14$ & 5.00 \\
\hline & \multicolumn{8}{|c|}{$\alpha_{\theta} \equiv 1, \alpha_{T} \equiv 0, \tau \equiv 1$} \\
\hline 3 & $9.75 \mathrm{E}-02$ & 0.98 & $4.62 \mathrm{E}-04$ & 2.95 & $5.49 \mathrm{E}-07$ & 4.31 & $3.11 \mathrm{E}-09$ & 4.98 \\
\hline 4 & $4.92 \mathrm{E}-02$ & 0.99 & $5.88 \mathrm{E}-05$ & 2.97 & $3.20 \mathrm{E}-08$ & 4.10 & $9.80 \mathrm{E}-11$ & 4.99 \\
\hline 5 & $2.47 \mathrm{E}-02$ & 0.99 & 7.43E-06 & 2.99 & $1.96 \mathrm{E}-09$ & 4.03 & $3.07 \mathrm{E}-12$ & 5.00 \\
\hline \multirow[t]{2}{*}{6} & $1.24 \mathrm{E}-02$ & 1.00 & $9.33 \mathrm{E}-07$ & 2.99 & $1.22 \mathrm{E}-10$ & 4.01 & $9.59 \mathrm{E}-14$ & 5.00 \\
\hline & \multicolumn{8}{|c|}{$\alpha_{\theta} \equiv 0, \alpha_{T} \equiv 1, \tau \equiv 1$} \\
\hline 3 & $1.43 \mathrm{E}-01$ & 0.94 & $3.79 \mathrm{E}-04$ & 2.94 & $5.77 \mathrm{E}-07$ & 4.60 & $1.75 \mathrm{E}-09$ & 4.96 \\
\hline 4 & $7.29 \mathrm{E}-02$ & 0.97 & $4.87 \mathrm{E}-05$ & 2.96 & $2.92 \mathrm{E}-08$ & 4.30 & $5.52 \mathrm{E}-11$ & 4.99 \\
\hline 5 & $3.68 \mathrm{E}-02$ & 0.98 & $6.17 \mathrm{E}-06$ & 2.98 & $1.70 \mathrm{E}-09$ & 4.10 & $1.73 \mathrm{E}-12$ & 5.00 \\
\hline \multirow[t]{2}{*}{6} & $1.85 \mathrm{E}-02$ & 0.99 & $7.77 \mathrm{E}-07$ & 2.99 & $1.04 \mathrm{E}-10$ & 4.03 & $5.41 \mathrm{E}-14$ & 5.00 \\
\hline & \multicolumn{8}{|c|}{$\alpha_{\theta} \equiv 1, \alpha_{T} \equiv 1, \tau \equiv 0$} \\
\hline 3 & $5.56 \mathrm{E}-02$ & 0.57 & $1.07 \mathrm{E}-04$ & 3.16 & $3.46 \mathrm{E}-07$ & 4.31 & $2.16 \mathrm{E}-09$ & 5.01 \\
\hline 4 & $3.60 \mathrm{E}-02$ & 0.62 & $1.25 \mathrm{E}-05$ & 3.10 & $1.99 \mathrm{E}-08$ & 4.12 & $6.73 \mathrm{E}-11$ & 5.00 \\
\hline 5 & $2.16 \mathrm{E}-02$ & 0.74 & $1.51 \mathrm{E}-06$ & 3.05 & $1.21 \mathrm{E}-09$ & 4.04 & $2.10 \mathrm{E}-12$ & 5.00 \\
\hline \multirow[t]{2}{*}{6} & $1.21 \mathrm{E}-02$ & 0.84 & $1.84 \mathrm{E}-07$ & 3.03 & $7.54 \mathrm{E}-11$ & 4.01 & $6.56 \mathrm{E}-14$ & 5.00 \\
\hline & \multicolumn{8}{|c|}{$\alpha_{\theta} \equiv 1, \alpha_{T} \equiv 1, \tau \equiv 1$} \\
\hline 3 & $5.42 \mathrm{E}-02$ & 0.89 & $1.74 \mathrm{E}-04$ & 2.87 & $5.20 \mathrm{E}-07$ & 4.33 & $2.76 \mathrm{E}-09$ & 4.97 \\
\hline 4 & $2.95 \mathrm{E}-02$ & 0.88 & $2.29 \mathrm{E}-05$ & 2.93 & $2.99 \mathrm{E}-08$ & 4.12 & $8.68 \mathrm{E}-11$ & 4.99 \\
\hline 5 & $1.59 \mathrm{E}-02$ & 0.89 & $2.94 \mathrm{E}-06$ & 2.96 & $1.83 \mathrm{E}-09$ & 4.03 & $2.72 \mathrm{E}-12$ & 5.00 \\
\hline 6 & $8.41 \mathrm{E}-03$ & 0.92 & 3.72E-07 & 2.98 & 1.14E-10 & 4.01 & $8.50 \mathrm{E}-14$ & 5.00 \\
\hline
\end{tabular}


TABLE 6.2. History of convergence of the numerical traces.

\begin{tabular}{|c|c|c|c|c|c|c|c|c|}
\hline \multirow[b]{2}{*}{ mesh } & \multicolumn{2}{|c|}{$k=0$} & \multicolumn{2}{|c|}{$k=1$} & \multicolumn{2}{|c|}{$k=2$} & \multicolumn{2}{|c|}{$k=3$} \\
\hline & $\|\widehat{e}\|_{\infty}$ & order & $\|\widehat{e}\|_{\infty}$ & order & $\|\widehat{e}\|_{\infty}$ & order & $\|\widehat{e}\|_{\infty}$ & order \\
\hline & \multicolumn{8}{|c|}{$\alpha_{\theta} \equiv 0, \alpha_{T} \equiv 0, \tau \equiv 1$} \\
\hline 3 & $1.43 \mathrm{E}-01$ & 0.96 & $4.33 \mathrm{E}-04$ & 2.95 & $2.36 \mathrm{E}-07$ & 4.99 & $3.21 \mathrm{E}-11$ & 6.98 \\
\hline 4 & $7.23 \mathrm{E}-02$ & 0.98 & $5.52 \mathrm{E}-05$ & 2.97 & 7.42E-09 & 4.99 & $2.49 \mathrm{E}-13$ & 7.05 \\
\hline 5 & $3.64 \mathrm{E}-02$ & 0.99 & $6.97 \mathrm{E}-06$ & 2.99 & $2.33 \mathrm{E}-10$ & 5.00 & $1.95 \mathrm{E}-15$ & 7.00 \\
\hline \multirow[t]{2}{*}{6} & $1.83 \mathrm{E}-02$ & 1.00 & $8.76 \mathrm{E}-07$ & 2.99 & $7.29 \mathrm{E}-12$ & 5.00 & $1.52 \mathrm{E}-17$ & 7.00 \\
\hline & \multicolumn{8}{|c|}{$\alpha_{\theta} \equiv 1, \alpha_{T} \equiv 0, \tau \equiv 1$} \\
\hline 3 & $1.01 \mathrm{E}-01$ & 0.97 & $4.33 \mathrm{E}-04$ & 2.95 & $2.36 \mathrm{E}-07$ & 4.99 & $3.21 \mathrm{E}-11$ & 6.98 \\
\hline 4 & $5.10 \mathrm{E}-02$ & 0.99 & $5.52 \mathrm{E}-05$ & 2.97 & $7.42 \mathrm{E}-09$ & 4.99 & $2.43 \mathrm{E}-13$ & 7.04 \\
\hline 5 & $2.56 \mathrm{E}-02$ & 0.99 & $6.97 \mathrm{E}-06$ & 2.99 & $2.33 \mathrm{E}-10$ & 5.00 & $1.91 \mathrm{E}-15$ & 6.99 \\
\hline \multirow[t]{2}{*}{6} & $1.28 \mathrm{E}-02$ & 1.00 & $8.76 \mathrm{E}-07$ & 2.99 & $7.29 \mathrm{E}-12$ & 5.00 & $1.50 \mathrm{E}-17$ & 6.99 \\
\hline & \multicolumn{8}{|c|}{$\alpha_{\theta} \equiv 0, \alpha_{T} \equiv 1, \tau \equiv 1$} \\
\hline 3 & $1.42 \mathrm{E}-01$ & 0.97 & $3.58 \mathrm{E}-04$ & 2.94 & $3.84 \mathrm{E}-07$ & 4.97 & $9.76 \mathrm{E}-12$ & 6.92 \\
\hline 4 & $7.17 \mathrm{E}-02$ & 0.98 & 4.60E-05 & 2.96 & $1.21 \mathrm{E}-08$ & 4.99 & $7.73 \mathrm{E}-14$ & 6.98 \\
\hline 5 & $3.61 \mathrm{E}-02$ & 0.99 & $5.84 \mathrm{E}-06$ & 2.98 & $3.80 \mathrm{E}-10$ & 4.99 & $6.08 \mathrm{E}-16$ & 6.99 \\
\hline \multirow[t]{2}{*}{6} & $1.81 \mathrm{E}-02$ & 1.00 & $7.36 \mathrm{E}-07$ & 2.99 & $1.19 \mathrm{E}-11$ & 5.00 & $4.78 \mathrm{E}-18$ & 6.99 \\
\hline & \multicolumn{8}{|c|}{$\alpha_{\theta} \equiv 1, \alpha_{T} \equiv 1, \tau \equiv 0$} \\
\hline 3 & $5.30 \mathrm{E}-02$ & 0.58 & $8.87 \mathrm{E}-05$ & 3.22 & $1.48 \mathrm{E}-07$ & 4.95 & $4.21 \mathrm{E}-11$ & 6.98 \\
\hline 4 & $3.43 \mathrm{E}-02$ & 0.63 & $1.01 \mathrm{E}-05$ & 3.14 & 4.70E-09 & 4.98 & $3.31 \mathrm{E}-13$ & 6.99 \\
\hline 5 & $2.06 \mathrm{E}-02$ & 0.74 & $1.19 \mathrm{E}-06$ & 3.08 & $1.48 \mathrm{E}-10$ & 4.99 & $2.60 \mathrm{E}-15$ & 6.99 \\
\hline \multirow[t]{2}{*}{6} & $1.15 \mathrm{E}-02$ & 0.84 & $1.44 \mathrm{E}-07$ & 3.04 & $4.64 \mathrm{E}-12$ & 4.99 & $2.03 \mathrm{E}-17$ & 7.00 \\
\hline & \multicolumn{8}{|c|}{$\alpha_{\theta} \equiv 1, \alpha_{T} \equiv 1, \tau \equiv 1$} \\
\hline 3 & $5.32 \mathrm{E}-02$ & 0.92 & $1.48 \mathrm{E}-04$ & 2.84 & $2.36 \mathrm{E}-07$ & 4.97 & $4.04 \mathrm{E}-12$ & 6.88 \\
\hline 4 & $2.81 \mathrm{E}-02$ & 0.92 & $1.97 \mathrm{E}-05$ & 2.91 & 7.44E-09 & 4.99 & $3.24 \mathrm{E}-14$ & 6.96 \\
\hline 5 & $1.49 \mathrm{E}-02$ & 0.92 & $2.53 \mathrm{E}-06$ & 2.96 & $2.33 \mathrm{E}-10$ & 4.99 & $2.57 \mathrm{E}-16$ & 6.98 \\
\hline 6 & $7.75 \mathrm{E}-03$ & 0.94 & $3.22 \mathrm{E}-07$ & 2.98 & $7.31 \mathrm{E}-12$ & 4.99 & $2.02 \mathrm{E}-18$ & 6.99 \\
\hline
\end{tabular}

\section{CONCLUDing REMARKS}

We have shown that optimal HDG methods can be devised which are free from shear-locking. We achieved this by a careful study of the relation between the definition of the numerical traces and the corresponding convergence properties of the methods. Key to our analysis was a new projection operator which is tailored to fit the structure of the numerical traces of the HDG method. We have shown that HDG solution superconverges to the projection of the exact solution for all the unknowns. This immediately results in optimal error estimates for all the unknowns. In this sense, the error analysis is simplified only to the study of the approximation properties of the projection operator.

This provides a powerful approach for devising locking-free HDG methods for more challenging problems arising in solid mechanics, like the Reissner-Mindlin plates problem. This constitutes the subject of ongoing work.

\section{Appendix A. Proof of the Regularity property OF THE DUAL PROBLEM}

The proof is based on the following simple lemma.

Lemma A.1. Let $f \in L^{2}(\Omega)$ and set $f^{n}(x):=\int_{0}^{x} f^{n-1}(t) d t$, for $n \geq 1$ with $f^{0}:=f$. Then $\left\|f^{n}\right\| \leq\|f\|$. 
We are now ready to prove the elliptic regularity inequality (2.7). A straightforward computation shows that the solution of (2.6) is given by

$$
\begin{aligned}
\psi_{T}= & -\eta_{\mathfrak{W}}^{1}+c_{T}, \quad \psi_{M}=-\eta_{\mathfrak{W}}^{2}+\eta_{\theta}^{1}+c_{T} x+c_{M}, \\
\psi_{\theta}= & \int_{0}^{x} \frac{1}{E I}\left(-\eta_{\mathfrak{W}}^{2}+\eta_{\theta}^{1}+c_{T} t+c_{M}\right) d t-\eta_{M}^{1}+c_{\theta}, \\
\psi_{\mathcal{W}}= & \int_{0}^{x} \int_{0}^{t} \frac{1}{E I}\left(-\eta_{\mathfrak{W}}^{2}+\eta_{\theta}^{1}+c_{T} s+c_{M}\right) d s d t \\
& +\int_{0}^{x} \frac{d^{2}}{G A} \eta_{\mathfrak{W}}^{1} d t-\eta_{M}^{2}+c_{\theta} x+c_{\mathfrak{W}} .
\end{aligned}
$$

where $c_{t}, c_{M}, c_{\theta}$, and $c_{w}$ are constants of integration. Using the boundary conditions $\psi_{\theta}(0)=\psi_{\mathcal{W}}(0)=0$, we immediately get $c_{\theta}=c_{\mathcal{W}}=0$. The remaining boundary conditions, $\psi_{\theta}(1)=\psi_{\mathfrak{w}}(1)=0$, yield the following linear system for $c_{T}$ and $c_{M}$ :

$$
\left(\begin{array}{ll}
a_{11} & a_{12} \\
a_{12} & a_{22}
\end{array}\right)\left(\begin{array}{c}
c_{T} \\
c_{M}
\end{array}\right)=\left(\begin{array}{l}
b_{1} \\
b_{2}
\end{array}\right)
$$

where

$$
a_{11}=\int_{0}^{1} \frac{1}{E I} t d t, a_{12}=\int_{0}^{1} \frac{1}{E I} d t, a_{21}=\int_{0}^{1} \int_{0}^{t} \frac{1}{E I} s d s d t, a_{22}=\int_{0}^{1} \int_{0}^{t} \frac{1}{E I} d s d t
$$

and $b_{1}=\int_{0}^{1} \frac{1}{E I}\left(\eta_{\mathfrak{w}}^{2}-\eta_{\theta}^{1}\right) d t+\int_{0}^{1} \eta_{M} d t$ and

$$
b_{2}=\int_{0}^{1} \int_{0}^{t} \frac{1}{E I}\left(\eta_{w}^{2}-\eta_{\theta}^{1}\right) d s d t-\int_{0}^{1} \frac{d^{2}}{G A} \eta_{w}^{1} d t+\int_{0}^{1} \eta_{M}^{1} d t
$$

By Lemma A.1 we can easily show that $\left|b_{i}\right| \leq C\|\eta\|$ for $i=1$, 2 , where the constant $C$ depends solely on $E I$ and $G A$. Note that the dependence on $d$ can be suppressed since $d \leq 1$. Furthermore, we can also suppress the dependence of $C$ on $E I$ and $G A$ since these, and hence their reciprocals, are functions of order one on $\Omega$. Thus, we have that $\left|c_{T}\right| \leq C\|\eta\|$, and that $\left|c_{M}\right| \leq C\|\eta\|$. Inserting these estimates into (A.1) and applying Lemma A.1 once more, we finally obtain (2.7).

\section{REFERENCES}

[1] F. Celiker, Discontinuous Galerkin methods for Structural Mechanics, Ph.D. thesis, University of Minnesota, 2005.

[2] F. Celiker and B. Cockburn, Element-by-element post-processing of discontinuous Galerkin methods for Timoshenko beams, J. Sci. Comput. 27 (2006), 177-187. MR2285774 (2007i:74042)

[3] F. Celiker, B. Cockburn, S. Güzey, R. Kannapady, S.-C. Soon, H. K. Stolarski, and K. K. Tamma, Discontinuous Galerkin methods for Timoshenko beams, Numerical Mathematics and Advanced Applications, ENUMATH 2003, Springer, 2003, pp. 221-231.

[4] F. Celiker, B. Cockburn and K. Shi, Hybridizable discontinuous Galerkin methods for Timoshenko beams, J. Sci. Comput. 44 (2010), 1-37 MR2647497

[5] F. Celiker, B. Cockburn, and H. Stolarski, Locking-free optimal discontinuous Galerkin methods for Timoshenko beams, SIAM J. Numer. Anal. 44 (2006), 2297-2325. MR2272595 (2007m:65108)

[6] B. Cockburn, J. Gopalakrishnan, and R. Lazarov, Unified hybridization of discontinuous Galerkin, mixed and continuous Galerkin methods for second order elliptic problems, SIAM J. Numer. Anal. 47 (2009), 1319-1365. MR2485455(2010b:65251) 
[7] B. Cockburn, J. Gopalakrishnan, and F.-J. Sayas, A projection-based error analysis of HDG methods, Math. Comp. 79 (2010), 1351-1367. MR2629996

[8] S. P. Timoshenko, On the correction for shear of the differential equation for transverse vibrations of prismatic bar, Philosophical Magazine 41 (1921), 744-746.

Department of Mathematics, Wayne State University, Detroit, Michigan 48202

E-mail address: celikern@math.wayne.edu

School of Mathematics, University of Minnesota, 206 Church Street S.E., MinneapoLIS, Minnesota 55455

E-mail address: cockburn@math.umn.edu

School of Mathematics, University of Minnesota, 206 Church Street S.E., MinneapoLis, Minnesota 55455

E-mail address: shixx075@math.umn.edu 